\title{
Zweites Kapitel: Erste Schritte nach dem Ende der nationalsozialistischen Herrschaft - Fürsorge und Rehabilitierung (1945-1947)
}

\section{Das Pariser Reparationsabkommen und die nichtrepatriierbaren Flüchtlinge}

Die USA hatten während des Krieges keinen Versuch gemacht, die Problematik der Entschädigung von Verfolgten des Nationalsozialismus im Zusammenhang der Reparationsfrage mit ihren Alliierten zu diskutieren. Das lag vor allem daran, daß kein inneramerikanischer Konsens in dieser Frage erreicht worden war. Erst kurz nach Kriegsende wurde ein Anlauf unternommen, der bezeichnenderweise an der Frage der von den Nationalsozialisten vertriebenen staatenlosen Flüchtlinge ansetzte, die in der US-Administration bei der Herausbildung eines Problembewußtseins hinsichtlich der nationalsozialistischen Verfolgung eine Schlüsselstellung eingenommen hatte.

In Jalta hatten sich Großbritannien, die Sowjetunion und die USA auf das Prinzip der deutschen Reparationspflicht geeinigt und einen gemeinsamen Ausschuß gegründet, der in Moskau die Einzelheiten der geplanten Regelung festlegen sollte. Zum Leiter der US-Delegation, die am 20. Mai 1945 nach Moskau aufbrach, bestimmte Präsident Truman Edwin W. Pauley, einen erfolgreichen Geschäftsmann und Makler ${ }^{1}$. Dieser erörterte vor seiner Abreise mit Truman auch die Möglichkeit, ein Abkommen anzustreben, wonach ein Teil der als Reparationen zu erbringenden Sachleistungen zugunsten staatenloser Personen, die von den Deutschen beraubt worden waren, verfügbar gemacht werden sollte. Der Präsident reagierte positiv auf diesen Vorschlag, hielt es aber noch für verfrüht, eine endgültige Entscheidung in dieser Angelegenheit zu treffen².

Nach dem weitgehenden Fehlschlag der Moskauer Reparationskonferenz wurde auf der Potsdamer Konferenz im Juli und August 1945 einen letztes Mal gemeinsam versucht, die strittig gebliebenen Fragen zu klären. Pauley, der wiederum die amerikanische Delegation leitete, verlor dort seinen früheren Gedanken nicht aus den Augen und entwickelte einen Vorschlag, der die staatenlosen Personen an den Reparationen beteiligen sollte ${ }^{3}$. Doch bei den Verhandlungen in Potsdam ergaben sich erneut Schwierigkeiten, weshalb es Pauley wiederum nicht opportun schien, seinen Vorschlag zu präsentieren. So hielt er seinen Entwurf zunächst zurück und schickte ihn erst Ende August an

1 Vgl. zu den Reparationsverhandlungen in Jalta, Moskau und Potsdam insbes. Otto Nübel, Die amerikanische Reparationspolitik gegenüber Deutschland 1941-1945, hrsg. v. Bundesministerium für Innerdeutsche Beziehungen, Frankfurt a.M. 1980, S. 158.

2 Edwin W. Pauley an Secretary of State James F. Byrnes, 29.8. 1945, Anlage zu Repmem 3, Reparation and Restitution for Victims of Nazi Persecution, 12.11. 1945, USNA, RG 43, Lot M 88, Box 11.

3 Pauley, August 1945, "Reparations and Restitution for Stateless Persons - Summary“, USNA, RG 43, Lot M 88 , Box 11. 
Außenminister Byrnes, in der Hoffnung, daß ihn dieser auf der bevorstehenden Londoner Außenministerkonferenz präsentieren könnte ${ }^{4}$.

Pauleys Memorandum ging von der gängigen Definition der Reparationen aus, wonach der besiegte Staat den Siegerstaaten eine Kriegsentschädigung zahlt. Die Ansprüche von Privatpersonen, deren Eigentum durch Deutschland vernichtet worden war, würden in der Regel durch ihre Regierungen vertreten. Doch gebe es niemanden, der die Ansprüche der Hunderttausenden von Menschen, die von Anfang an gegen Hitler gekämpft hätten und von den Nationalsozialisten ausgeraubt worden seien, verfechte, da diese staatenlos geworden seien. Deshalb schlug Pauley vor, eine internationale Kommission zu gründen, die anstelle einer Regierung die Interessen der staatenlosen Personen gegen Deutschland vertreten solle. Diese müßte einen bestimmten Anteil an den Reparationen erhalten und damit die Unterstützung, Rehabilitierung und Wiederansiedlung dieser staatenlosen Personen finanzieren ${ }^{5}$. Pauley zielte also auf solche Folgen der nationalsozialistischen Politik, die sich im Rahmen des traditionellen Völkerrechts, das auf souveräne Nationalstaaten abhob, nicht bewältigen ließen. Der Umstand, daß eine noch unabsehbar große, aber vermutlich in die Hunderttausende gehende Zahl von staatenlosen Menschen entwurzelt in der Welt herumirrte und vorhandene soziale Probleme verschärfte, hatte ja bereits seit den dreißiger Jahren für Beunruhigung gesorgt, das State Department seit 1943 zu Planungen angeregt und harrte nun einer Lösung.

Besonders interessiert an dieser Frage waren die internationalen jüdischen Organisationen. Der World Jewish Congress bildete im Oktober 1945 zusammen mit der Jewish Agency und der American Jewish Conference ein Komitee zu dem Zwecke, einen gemeinsamen jüdischen Reparationsanspruch zu erheben ${ }^{6}$. Den ersten wichtigen Schritt dazu hatte bereits Chaim Weizmann mit einem Schreiben an die vier Siegermächte vom 20. September unternommen, das auf den von Bernhard Joseph formulierten Argumenten beruhte ${ }^{7}$. Weizmann forderte, daß das jüdische Volk bei den bevorstehenden Verhandlungen über die Aufteilung der von Deutschland zu zahlenden Reparationen berücksichtigt werden müsse. Der ihm zukommende Anteil sollte der Jewish Agency anvertraut werden, die damit die Rehabilitierung und Ansiedlung jüdischer Opfer des NS-Regimes in Palästina betreiben sollte ${ }^{8}$. Weizmann konzentrierte sich in diesem Schreiben auf die kollektiven jüdischen Forderungen. Zugleich kündigte er aber für die Zukunft auch gemeinsame Schritte der jüdischen Organisationen in der Frage der individuellen Forderungen nach Rückerstattung und Entschädigungen an.

Am 19. Oktober traf eine Delegation des gemeinsamen jüdischen Komitees unter Führung Nahum Goldmanns im State Department mit Staatssekretär Dean Acheson und einigen anderen Beamten zusammen, um den Brief Weizmanns persönlich zu übergeben. Goldmann unterstrich, daß sie als Vertreter des gesamten jüdischen Volkes sprächen und insbesondere die staatenlosen europäischen Juden verträten. Auf Nachfrage erklärte Goldmann, staatenlos sei auch jeder, der nicht mehr heimkehren wolle.

4 Ebenda.

5 Ebenda.

6. Vgl. dazu auch Leon Kubowitzki, Unity in Dispersion, A History of the World Jewish Congress, New York 1948 , S. $270 \mathrm{f}$.

7 Vgl. dazu Erstes Kapitel, Abschnitt II. 4.

${ }^{8}$ Chaim Weizmann to the Secretary of State, 20.9. 1945, in: Foreign Relations of the United States (FRUS), 1945, Bd. III: European Advisory Commission, Austria, Germany, Washington, D.C., 1968, S. 1302-1305. 
Das bezog sich vor allem auch auf diejenigen polnischen Juden, denen die Staatsbürgerschaft zwar nicht aberkannt worden war, die aber nicht mehr in ihrer ehemaligen Heimat leben wollten. Goldmann unterschied dabei zwei Kategorien von Ansprüchen, nämlich einmal Rückerstattung und Entschädigung und zum anderen Reparationen. Bei ersteren ginge es insbesondere um den geraubten jüdischen Besitz, der einschließlich des nunmehr erbenlosen Vermögens Eigentum der jüdischen Nation sei. Hier beschränkte sich Goldmann im Anschluß an Weizmann zunächst auf die Ankündigung späterer Initiativen. Den unmittelbaren Anlaß für seinen Besuch bildete hingegen der Wunsch, im Rahmen der gegenwärtig laufenden Verhandlungen der Siegermächte über die Aufteilung der Reparationen berücksichtigt zu werden?.

Parallel dazu wandte sich Jacob Blaustein vom American Jewish Committee an US-Außenminister Byrnes. Blaustein war eine der zentralen jüdischen Figuren im Kampf um die Wiedergutmachung. Er stützte sich auf ein bedeutendes Wirtschaftsimperium im Bereich der Erdölindustrie sowie auf erstklassige politische Kontakte in Washington. Ihm standen die Türen zu den obersten Etagen der US-Administration, einschließlich des Präsidenten, offen ${ }^{10}$. Blaustein wies Byrnes gleichfalls auf das Problem hin, daß deutsche sowie staatenlose und nichtrepatriierbare Juden - so wie die Dinge jetzt lägen - keine Ansprüche geltend machen könnten und mahnte „eine kollektive Verantwortung der Vereinten Nationen" 11 für diese Menschen an. Er schlug vor, einen bestimmten Prozentsatz der deutschen Reparationen abzuzweigen und von einer Treuhandschaft der Vereinten Nationen verwalten zu lassen. Diese „United Nations Trusteeship of Indemnifications“ - also kein explizit jüdisches Kollektiv - sollte als Statthalter der Reparationsansprüche deutscher, staatenloser und nichtrepatriierbarer Juden auftreten. Damit standen seine Forderungen in einem gewissen Gegensatz zu denen Nahum Goldmanns, dem Repräsentanten der Jewish Agency. Blausteins Vorschläge bewegten sich stärker auf der Linie, die bereits Pauley in der Diskussion der US-Administration eingeschlagen hatte, allerdings beschränkt auf jüdische Verfolgte.

Auch im State Department wurde der Gedanke von Reparationen für die staatenlosen Verfolgten vor allem entlang der Vorschläge Pauleys weiterentwickelt. Dabei lockte das State Department insbesondere auch die Aussicht, durch einen Anteil an den Reparationen eine unabhängige, nichtamerikanische Finanzierungsquelle für die Hilfsaktionen des Inter-Governmental Committee on Refugees zugunsten deutscher und österreichischer nichtrepatriierbarer Personen zu gewinnen ${ }^{12}$. Als im Oktober James W. Angell neuer Vorsitzender der US-Delegation bei der alliierten Reparationskommission wurde, beauftragte ihn Byrnes in seiner Ernennung auch ausdrücklich damit, „bei den Treffen der alliierten Reparationskommission in Paris die Auf-

9 Department of State. Memorandum of Conversation, Nahum Goldmann, Maurice Perlzweig, Dean Acheson u. a. 19. 10. 1945, USNA, RG 59, 740.00119 EW/9-2045.

$10 \mathrm{Vgl}$. Brief Biographic Scetch of Jacob Blaustein, 1.12. 1967, AJC-Archiv, Blaustein Library, Restitution \& Indemnification.

11 Blaustein an Byrnes, 17. 10. 1946, YIVO-Archiv, RG 347, AJC-Records, GEN-10, Box 281. Zu den Bemühungen Blausteins und des American Jewish Committee vgl. auch AJC Contributions to Postwar Economic Rehabilitation of Jewish Victims of Nazi Persecution, September 1965, AJC-Archiv, JSX, Subject Restitution 65-66.

12 War Department an OMGUS, 23. 8. 1946, IfZ-Archiv, MF 260, OMGUS, AG 1948/184/1. Vgl. auch Ronald Zweig, German Reparations and the Jewish World: A History of the Claims Conference, Boulder u. London 1987, S.3. 
fassung der Vereinigten Staaten bezüglich Reparationen und Rückerstattung für die deutschen Opfer der Nazi-Verfolgung zu vertreten" ${ }^{13}$.

In Paris verhandelten 18 alliierte Staaten vom 9. November bis 21. Dezember über die Aufteilung der Reparationsanteile aus den westlichen Besatzungszonen. Bei dieser Gelegenheit versuchte die US-Delegation nunmehr nachdrücklich, einen Anteil für staatenlose, nichtrepatriierbare Verfolgte des Nationalsozialismus zu erreichen. Im Reisegepäck führte sie ein Memorandum mit, worin die allgemeinen Grundsätze der Entschädigung für aus rassischen, religiösen oder politischen Gründen verfolgte Personen festgelegt waren ${ }^{14}$. In der Substanz handelte es sich dabei um eine Präzisierung der Vorschläge Pauleys. So sollte eine internationale Behörde geschaffen werden, die einen Anteil an den Reparationen erhalten und zugunsten der großen Zahl von Verfolgten, die durch keine Regierung vertreten würden, zu verteilen hätte. Die Verfolgten wurden hier in mehrere Gruppen eingeteilt: Die erste Gruppe bildeten demnach diejenigen Verfolgten, die in Deutschland geblieben waren oder dorthin zurückkehren würden. Die Entschädigung für diesen Kreis galt als ein inneres deutsches Problem, mit dem sich in erster Linie der Alliierte Kontrollrat und die Zonenkommandeure zu befassen hätten. In diesem Zusammenhang wurde auch darauf verwiesen, daß Eisenhower angewiesen worden sei, sich um eine schleunige Rückerstattung entzogener Vermögen zu bemühen. Dieser Gruppe wurden drei andere gegenübergestellt: Verfolgte aus ehemals vom Deutschen Reich besetzten Ländern, nicht rückkehrwillige Flüchtlinge aus Deutschland sowie staatenlose Personen im allgemeinen. Letztere besäßen ohnehin keine Regierung, die ihre Ansprüche vertreten würde, doch auch unter den anderen gäbe es viele, die aus politischen oder anderen Motiven nicht mehr in ihre Heimat zurückkehren wollten. Das hieß, eine große Zahl von Opfern des NS-Regimes war ohne Vertretung durch eine Regierung und deshalb in gewisser Hinsicht der vorgeschlagenen internationalen Behörde schutzbefohlen.

Auch diese Überlegungen fußten auf dem brennenden Problem der Existenz einer großen entwurzelten Menschengruppe ohne Daseinsgrundlage, die bedürftig der Völkergemeinschaft zur Last fiel. Deshalb war hier erneut das Prinzip festgehalten worden, $\mathrm{daß}$ „Reparationen, die diese Behörde erhalten würde, nur denjenigen zukommen sollten, die Hilfe bei ihrer Rehabilitierung oder Wiederansiedlung benötigten, und nicht auf der Grundlage irgendeines Rechtsanspruchs auf Entschädigung verteilt werden sollten " ${ }^{15}$. Zur Finanzierung dieser Behörde schlug das Memorandum vor, erstens einen bestimmten Prozentsatz der Reparationen (etwa ein oder zwei Prozent) und zweitens das nichtmonetäre Gold, Edelsteine usw., das die Nationalsozialisten ihren Opfern geraubt hatten, zu verwenden.

Am 13. November übergab US-Delegationsleiter Angell der britischen und französischen Delegation eine Zusammenfassung des US-Standpunkts über die Zuteilung eines Anteils an den deutschen Reparationen für heimatlose Opfer Nazi-Deutschlands ${ }^{16}$. Angell klärte die Sache erst mit den Briten ab, bevor er das Projekt dem Plenum vorlegte. Diese reagierten zunächst zurückhaltend, hätten sie es doch offensichtlich

13 Byrnes an James W. Angell, 24. 10. 1945, in: FRUS 1945 III, S. 1359.

14 Siehe zum folg. Repmem 3: Reparation and Restitution for Victims of Nazi Persecution, 12. 11. 1946, USNA RG 43, Lot M 88, Box 11.

15 Ebenda.

16 Angell, ,Note on Allocating a Share of German Reparations for Displaced Victims of Nazi Germany“, 13.11. 1945, USNA, RG 59, 740.00119 EW/9-2045. 
lieber gesehen, wenn die Flüchtlinge wieder in ihre Heimatländer zurückkehrten, statt nun Unterstützung zu beanspruchen und am Ende auch noch die britische PalästinaPolitik in Schwierigkeiten zu bringen ${ }^{17}$.

Heftige, anders begründete Kritik übten am 30. November Gray und Gottschalk, zwei Vertreter des American Jewish Committee. Sie unterbreiteten Angell eine Reihe von Wünschen, die vom State Department auch geprüft wurden, aber keinen besonderen Eindruck hinterließen. Immerhin aber stimmte das State Department ihrer Auffassung $z u, d a ß$ es sich bei der eingeleiten Aktion nur um eine vorläufige Maßnahme handele ${ }^{18}$. Auch der Jüdische Weltkongreß war durch Nehemiah Robinson, der sich dort als Beobachter aufhielt, in Paris vertreten ${ }^{19}$. Doch gelang es den jüdischen Organisationen insgesamt nicht, irgendeinen nennenswerten Einfluß auf Verlauf und Ergebnisse dieser Konferenz zu nehmen.

Bevor der amerikanische Entwurf am 10. Dezember dem Plenum der Pariser Konferenz vorgelegt wurde, hatte er bereits eine Reihe von Modifikationen durchlaufen, die vor allem auf britische Intervention zurückgingen ${ }^{20}$. Die anschließenden Verhandlungen verliefen zäh. Ausdauernd wurde um die Höhe der Leistungen und die genaue Definition der Anspruchsberechtigten gerungen. So wollten etwa die französischen, jugoslawischen und tschechoslowakischen Delegationen auch die republikanischen Spanien-Flüchtlinge in diese Regelung einbezogen wissen, was aber das State Department strikt ablehnte ${ }^{21}$. Schließlich setzten sich die amerikanischen Grundvorstellungen weitgehend durch. Artikel 8 der Pariser Vereinbarungen vom 21. Dezember 1945 enthielt die Bestimmung, daß alles von den alliierten Armeen in Deutschland aufgefundene ungemünzte Gold (dahinter verbargen sich die den ermordeten Opfern abgenommenen Wert- und Schmucksachen) für die Rehabilitierung und Wiederansiedlung nichtrepatriierbarer Opfer des Nationalsozialismus verwendet werden sollte. Als weitere Finanzierungsquellen für diesen Zweck nannte das Abkommen die deutschen Auslandsvermögen in Schweden, der Schweiz und Portugal, aus denen man einen Betrag von 25 Mio. Dollar abzuzweigen wünschte, sowie das erbenlose Eigentum verstorbener Verfolgter des Nationalsozialismus in neutralen Staaten ${ }^{22}$.

Ausdrücklich wurde darauf verwiesen, daß der Fonds nicht zur Befriedigung individueller Entschädigungsansprüche, sondern zur Rehabilitierung und Wiederansiedelung „echter Opfer der NS-Verfolgung “23 und ihrer engsten Angehörigen gedacht war. Darunter fielen Flüchtlinge aus Deutschland und Österreich (in besonderen Fällen auch solche, die erst noch aus Deutschland auswandern wollten) sowie Angehörige der vom Deutschen Reich besetzten Länder, sofern sie in einem KZ inhaftiert gewesen waren. Dafür enthielt das Abkommen aber auch die explizite Bestimmung, daß dadurch keine

17 Britische Antwort vom 19.11. 1945 und US-Kommentar, Jefferson Caffery (US-Botschafter in Paris) an Byrnes, 2. 12. 1945, in: FRUS 1945 III, S. 1437f.

18 Byrnes an Caffery, 10.12. 1945, in: FRUS 1945 III, S. 1452.

19 Vgl. Nana Sagi, Wiedergutmachung für Israel. Die deutschen Zahlungen und Leistungen, Stuttgart 1981, S. 40.

20 US Reparations Delegation, CPR/Doc. 48, 7.12. 1945, Paris Conference on Reparations. „Proposed Recommendation of Reparation Share for Non-Repatriable Victims of German Action“, USNA, RG 43, Lot M 88, Box 11.

${ }^{21}$ Siehe dazu insbesondere FRUS 1945 III, S. 1454-1475; sowie Material in USNA, RG 43, Lot M 88, Box 11.

22 Final Act and Annex of the Paris Conference on Reparation, Part I, Art. 8: „Allocation of a Reparation Share to Non-Repatriable Victims of German Action“, in: Department of State Bulletin, Bd. XIV, Nr. 343, 27.1. 1946, S. $118 f$.

${ }^{23} \mathrm{Vgl}$. ebenda. 
künftigen individuellen Ansprüche gegen das Deutsche Reich geschmälert würden. Erneut wurde damit deutlich gemacht, daß es hier primär um Sofortmaßnahmen zur Eindämmung des infolge der nationalsozialistischen Politik entstandenen Flüchtlingsproblems ging und weniger um die Wiederherstellung geschädigter Rechte.

Die genauen Modalitäten der Verteilung und Verwaltung dieser Mittel wurden erst auf einer weiteren Konferenz der Regierungen der Vereinigten Staaten, Großbritanniens, Frankreichs, der Tschechoslowakei und Jugoslawiens mit dem Intergovernmental Committee for Refugees im Juni 1946 in Paris festgelegt ${ }^{24}$. Am 14. Juni unterzeichneten die fünf Regierungen ein Abkommen, worin Artikel 8 des Pariser Abkommens unter anderem dahingehend präzisiert wurde, daß 90 Prozent der Beträge für jüdische Flüchtlinge ausgegeben werden sollten, da sie den Großteil der nichtrepatriierbaren Flüchtlinge bildeten. Die für die nichtjüdischen Betroffenen verfügbaren 10 Prozent sollten durch das Intergovernmental Committee for Refugees oder eine entsprechende Nachfolgeorganisation verwaltet werden. Der für die Rehabilitierung und Wiederansiedlung jüdischer Flüchtlinge zur Verfügung stehende Betrag kam hingegen dem American Joint Distribution Committee und der Jewish Agency for Palestine für ihre Hilfstätigkeit $\mathrm{zu}^{25}$.

Damit hatten die jüdischen Organisationen einen Erfolg verbuchen können. Es war anerkannt worden, was ohnehin auf der Hand lag, daß nämlich die meisten der nichtrepatriierbaren Opfer des Nationalsozialismus Juden waren, daß sie ein Recht auf Unterstützung hatten und daß der American Joint und die Jewish Agency als die Vertreter des jüdischen Anspruches angesehen wurden. In ihrem Verständnis stellte dies die Anerkennung eines jüdischen Kollektivanspruches $\mathrm{dar}^{26}$. Aus der Perspektive der Vereinigten Staaten, die dieses Abkommen initiiert hatten, und der anderen Signatarmächte war der kollektive Charakter dieser Leistungen aber eigentlich eher eine Verlegenheitslösung, die nicht zuletzt dem geringen Umfang der zur Verfügung stehenden Mittel Rechnung trug. Durch die Konzentration der Mittel auf einige Hilfsorganisationen sollten sie möglichst effektiv zur Linderung der dringendsten Nöte der nichtrepatriierbaren Flüchtlinge beitragen. Die Grundintention war mehr auf fürsorgliche Beseitigung eines internationalen Mißstandes als auf Wiederherstellung geschädigter Rechte gerichtet.

Dieser Ast der Wiedergutmachung trug nur wenig Früchte. Es bereitete große Mühe, die in dem Abkommen festgelegten Beträge überhaupt einzutrieben. Sowohl die Verwertung des ungemünzten Goldes als auch Zahlungen aus dem deutschen Vermögen in den neutralen Ländern ließen lange Jahre auf sich warten. Überdies waren die ca. 30 Mio. Dollar, die unter Bezug auf das Abkommen insgesamt aufgebracht wurden, ohnehin nur ein Tropfen auf den heißen Stein ${ }^{27}$. Doch blieb das Pariser Abkommen auf mehrere Jahre hinaus der einzige Versuch, einen kollektiven Entschädigungsanspruch

24 Vgl. dazu Eli Ginzberg, Reparation for Non-Repatriables, in: Department of State Bulletin, Bd. XV, JulyDec. 1946, S. 56 u. 76; Schalom Adler-Rudel, Aus der Vorzeit der kollektiven Wiedergutmachung, in: In zwei Welten. Siegfried Moses zum 75. Geburtstag, hrsg. v. Hans Tramer, Tel Aviv 1962, S. 214 f.; Sagi, Wiedergutmachung für Israel, S. 40f.

25 Agreement Pertaining to Reparation Funds for Non-Repatriable Victims of German Action, 14.6. 1946, in: Department of State Bulletin, Vol. XV, July-Dec. 1946, S. 71-73.

26 Vgl. Adler-Rudel, Vorzeit der kollektiven Wiedergutmachung, S. 216; Sagi, Wiedergutmachung für Israel, S. 42.

27 Vgl. Adler-Rudel, Vorzeit der kollektiven Wiedergutmachung, S. 215 f.; Sagi, Wiedergutmachung für Israel, S. $41 \mathrm{f}$. 
zugunsten von Verfolgten des Nationalsozialismus durchzusetzen. Die unmittelbare Nachkriegssituation hatte für einige Zeit die letzte Gelegenheit geboten, Ansprüche gegen Deutschland als Ganzes zu stellen, waren doch gerade bei den Reparationsverhandlungen starke Gegensätze unter den Alliierten sichtbar geworden. Für eine Reihe von Jahren mußten alle Forderungen von der Tatsache ausgehen, daß Deutschland in Besatzungszonen geteilt war, die nur anfänglich mehr schlecht als recht durch den Alliierten Kontrollrat koordiniert waren.

\section{Alliierte und amerikanische Konzepte zur Betreuung der Ver- folgten des Nationalsozialismus in Deutschland}

\section{Vorbereitungen im European Advisory Committee}

Vor und während des Krieges und auch noch auf der Pariser Reparationskonferenz gelangten in erster Linie diejenigen Opfer des Nationalsozialismus, die aus dem Deutschen Reich geflohen waren, in den Problemhorizont der USA und ihrer Alliierten. Dies änderte sich jedoch, als die alliierte Besetzung Deutschlands bevorstand: Nun mußte die Frage beantwortet werden, was aus den Insassen der nationalsozialistischen Konzentrationslager und Gefängnisse auf deutschem Boden werden sollte. Aus dem grundsätzlichen Ziel der Zerschlagung des nationalsozialistischen Systems ergab sich so auch die Notwendigkeit, hier tätig zu werden. Dabei ging es zunächst nicht um die Entschädigung der an diesen Menschen begangenen Verbrechen, sondern um Schritte, die der Fürsorge und Rehabilitierung dienten. In diesem Kontext wurde die Definition der nationalsozialistischen Verfolgung und der davon betroffenen Gruppen, die bislang vor allem im Zusammenhang des Flüchtlingsproblems stand, fortentwickelt. Zudem ergaben sich hier bereits Ansatzpunkte für künftige weiterreichende Maßnahmen der materiellen Entschädigung.

Schon bei den Beratungen der European Advisory Commission in London seit Januar 1944, in denen es um eine gemeinsame Politik der Alliierten für die Zeit unmittelbar nach der deutschen Kapitulation ging, hatte dieses Problem immer wieder eine Rolle gespielt $^{28}$. Es kristallisierte sich bald ein alliierter Grundkonsens heraus, der einerseits die Befreiung der politischen Gefangenen in Deutschland, andererseits die Beseitigung von Diskriminierungen aus rassischen, religiösen, politischen und anderen Gründen einschloß. Die damit ins Auge gefaßten Maßnahmen waren schon für den Fall der Niederlage des faschistischen Italiens gefordert worden ${ }^{29}$ und trugen vorläufigen Charakter. Sie standen in engem Zusammenhang mit der angestrebten Zerschlagung der Machtgrundlagen der nationalsozialistischen Herrschaft und besaßen zunächst nur eine begrenzte Perspektive.

Nach mehrmonatigen Verhandlungen in London hatten sich die USA, Großbritannien und die Sowjetunion darauf geeinigt, einen gemeinsamen, verhältnismäßig knap-

${ }^{28}$ Zur European Advisory Commission vgl. Hans-Günter Kowalski, Die „European Advisory Commission“ als Instrument alliierter Deutschlandplanung 1943-1945, in: Vierteljahrshefte für Zeitgeschichte (VfZ) 19 (1971), S. 261-293.

29 Policy and Administration in Connection with the Military Operations in Italy, Joint Note by Mr. Hull and Mr. Eden, Moskau, 23. 10. 1943, in: FRUS 1943, Bd. I: General, Washington, D.C., 1963, S.715-719, hier: S. 716 . 
pen Entwurf für die bedingungslose Kapitulation Deutschlands zu verfassen, der später durch detaillierte Direktiven zur Regelung von Einzelfragen ergänzt werden sollte. In der EAC-Sitzung vom 25. Juli 1944 wurde er beschlossen ${ }^{30}$. Im Anschluß an eine Bestimmung über die Behandlung der alliierten Kriegsgefangenen regelte der Artikel 6(b), daß die deutschen Behörden in gleicher Weise auch alle anderen gefangenen Bürger der Vereinten Nationen freilassen sollten und für diese zu sorgen hätten. Eine entsprechende Regelung trafen die Alliierten hier aber auch zugunsten derjenigen Personen, die „aus politischen Gründen oder in Folge irgendwelcher NS-Handlungen, Gesetze oder Verordnungen, die aus Gründen der Rasse, des Glaubensbekenntnisses oder der politischen Überzeugung " diskriminierten, inhaftiert waren ${ }^{31}$. Damit war also ein knappes Jahr vor Kriegsende festgelegt, daß Verfolgte des Nationalsozialismus befreit werden und Anspruch auf Betreuung durch die deutschen Behörden haben sollten. Die alliierte Definition der nationalsozialistischen Verfolgung griff dabei die Kriterien auf, die sich im Zuge der Diskussion um das deutsche Flüchtlingsproblem herauskristallisiert hatten: rassische, religiöse und politische Motive der Verfolgung wurden hier zugrundegelegt.

Anstelle der zunächst geplanten detaillierten Direktiven zu einzelnen Punkten der Kapitulationserklärung entstand in der EAC schließlich eine allgemeine Anweisung, die die dort festgelegten Punkte präzisierte ${ }^{32}$. Nach langwierigen Verhandlungen unterzeichneten die Vertreter der - nach der zwischenzeitigen Aufnahme Frankreichs in den Kreis der Siegermächte - vier Delegationen am 25. Juli 1945 in London ein „Abkommen über die Auferlegung bestimmter zusätzlicher Forderungen an Deutschland“ ${ }^{33}$. Der Alliierte Kontrollrat verkündigte das Abkommen schließlich am 20. September als Proklamation Nr.2. Hier waren noch einmal die Abschaffung der diskriminierenden Gesetzgebung sowie das Verbot der Diskriminierung aus rassischen, religiösen und politischen Gründen bzw. wegen Unterstützung der Vereinten Nationen aufgeführt ${ }^{34}$. Doch ging die Anordnung in einer Beziehung bereits über die bisher genannten Maßnahmen hinaus. Absatz 42(b) bestimmte die Gültigkeit künftiger Anordnungen des Alliierten Kontrollrats in Bezug auf das Eigentum, die Rechte, Ansprüche und Interessen von rassisch, religiös und politisch Verfolgten ${ }^{35}$. Hier zeigte sich die gemeinsame Absicht der Alliierten, über die elementaren Maßnahmen zur Befreiung und Beseitigung der Diskriminierung von Verfolgten des Nationalsozialismus hinaus auch weiterführende Schritte zu deren Gunsten zu unternehmen.

30 Minutes of the Seventh Formal Meeting of the European Advisory Commission, London, 25. 7. 1944, in: FRUS 1944, Bd. I: General, Washington, D.C., 1971, S. 252-261, dort auch Text des „Unconditional Surrender of Germany“, S. 256-261. Auf diesem Text beruhte dann die am 12.5. 1945 veröffentlichte „Erklärung in Anbetracht der Niederlage Deutschlands und der Übernahme der obersten Regierungsgewalt hinsichtlich Deutschlands, in: Amtsblatt des Kontrollrats in Deutschland, 1945, Ergänzungsblatt Nr. 1, S. 7 ff.

31 Minutes of the Seventh Formal Meeting of the European Advisory Commission, London, 25. 7. 1944, in: FRUS 1944 I, S. 259.

32 Zur Geschichte der „Zusätzlichen Anordnungen“ vgl. insbesondere das Memorandum von Moseley v. 19.6. 1945, The "General Order" or Agreement on Additional Requirements, in: FRUS 1945 III, S. 524-526.

33 Siehe dazu Report on the Work of the EAC, 10.9. 1945, in: FRUS 1945 III, S. 554; Text des Abkommens in FRUS 1945II, Conference of Berlin (Potsdam), Washington, D.C., 1960, S. $1011 \mathrm{ff}$.

34 Amtsblatt des Kontrollrats in Deutschland, Nr. 1, 29.10. 1945, Abs. 42 a, 43, S. 8-19, hier: S. 18.

${ }^{35}$ Ebenda. 


\section{Koordinierungsversuche im Alliierten Kontrollrat}

Die ersten Maßnahmen des Alliierten Kontrollrats standen, soweit sie die Opfer des Nationalsozialismus betrafen, noch ganz im Zeichen der Diskussionen der EAC, deren Auflösung auf der Potsdamer Konferenz beschlossen worden war. Kontrollratsgesetz Nr. 1 verfügte am 20. September 1945 die Aufhebung aller aus rassischen, religiösen und politischen Gründen diskriminierenden NS-Gesetzgebung ${ }^{36}$. Diese Maßnahme, über die schon seit 1943 Übereinstimmung unter den Alliierten geherrscht hatte, war auch noch einmal ausdrücklich im Potsdamer Abkommen verankert worden ${ }^{37}$. Die Kontrollrats-Proklamation Nr.3 über Grundsätze für die Umgestaltung der Rechtspflege sah ergänzend die Aufhebung von Verurteilungen aus rassischen, religiösen und politischen Gründen vor ${ }^{38}$.

Tatsächlich wurde auf Kontrollratsebene über einige Zeit hinweg versucht, eine gemeinsame Politik hinsichtlich der Verfolgten auf deutschem Boden festzulegen. Doch gelangte diese insgesamt nicht über Fürsorgemaßnahmen hinaus. Die Beschäftigung mit diesen Fragen war dabei allerdings auf verschiedene Kontrollrats-Direktorate verteilt. Zum Beispiel wurde auf französischen Vorschlag Anfang 1946 über eine Steuerbefreiung für Verfolgte beraten, doch fand dies keine Zustimmung im zuständigen alliierten Finance Directorate ${ }^{39}$. Eine erfolgreiche Maßnahme des Kontrollrats zugunsten der Verfolgten war dagegen der Erlaß eines Wohnungsgesetzes am 8. März 1946: Gesetz Nr. 18 regelte einheitlich die Grundsätze der Wohnungsbewirtschaftung im Nachkriegsdeutschland, die unter dem doppelten Druck weitflächig zerstörten Wohnraumes und millionenfach zuströmender Menschen stand. Dabei wurde „Personen, die dem nationalsozialistischen Regime Widerstand geleistet haben oder durch seine Maßnahmen benachteiligt worden sind“, die erste Priorität bei der Vergabe freien Wohnraums durch die deutschen Wohnungsämter zugesprochen ${ }^{40}$.

Der weitreichendste Ansatz zu einer alliierten Regelung der Fürsorge für Verfolgte des Nationalsozialismus ging von amerikanischer Seite aus. Sein Schicksal ist symptomatisch für Versuche auf Kontrollratsebene, Maßnahmen zugunsten dieser Gruppe zu treffen, die zwangsläufig grundsätzliche Fragen der gesellschaftlichen Entwicklung des besetzten Deutschlands berührten. Im November 1945 legte die US-Delegation im Committee on Social Insurance des Kontrollrats einen ersten Entwurf für Grundsätze der Sozialversicherung für Opfer des Nationalsozialismus vor ${ }^{41}$. Darin wurde allen bedürftigen Verfolgten und ihren Angehörigen das Recht auf Fürsorge durch das deutsche Volk zugesprochen. Dazu sollten alle Verfolgten im Rahmen des bestehenden Sozialversicherungssystems automatisch und unabhängig von ihren tatsächlichen Beitragsleistungen anspruchsberechtigt werden. Eingeschlossen waren sowohl Kranken-, Renten-, Invaliditäts- und Arbeitslosenversicherungen. Die Verfolgten sollten jeweils

${ }^{36}$ Amtsblatt des Kontrollrats in Deutschland, Nr. 1, 29. 10. 1945, S. 6-8.

37 Vgl. Abschnitt III.A.4, in: Um den Frieden mit Deutschland. Dokumente zum Problem der deutschen Friedensordnung 1948, hrsg. v. W. Cornides u. H. Volle, Oberursel (Ts.) 1948, S. 81.

38 Amtsblatt des Kontrollrats in Deutschland, Nr. 1, 29. 10. 1945, S. 22 f.

39 Allied Control Authority (ACA), Directorate of Finance, Taxation Committee, DFIN/TC/MEMO (46)15, 24. 5. 1946, IfZ-Archiv, MF 260, OMGUS-ACA 2/101-3/6.

40 Kontrollratsgesetz Nr. 18 vom 8.3. 1946, in: Amtsblatt des Kontrollrats in Deutschland, Nr. 5, 31. 3. 1946, S. 117-121, hier S. 119.

41 Denis A. Cooper (OMGUS/Manpower Division) 26.11. 1945, „Basic Principles of Social Insurance for Nazi Victims", IfZ-Archiv, MF 260, OMGUS-CAD 5/323-3/6. 
den höchsten Satz beziehen. Das Social Insurance Committee übernahm den amerikanischen Entwurf im Januar 1946 mit nur geringen Änderungen in eine eigene Vorlage ${ }^{42}$. Doch in den Beratungen des übergeordneten Manpower Directorate am 19. Februar wurde bemängelt, daß der Entwurf zu weit ginge. Es wurde angeregt, das Problem besser im Zusammenhang der geplanten Neustrukturierung des gesamten deutschen Sozialversicherungssystems zu regeln. Überdies hielt es das Manpower Directorate für erforderlich, die dem Entwurf zugrundeliegende Definition der „Opfer des Nationalsozialismus" noch mit anderen Kontrollrats-Direktorien abzustimmen ${ }^{43}$.

Nun begann ein Prozeß der Verwässerung des ursprünglichen Konzepts zur Regelung der Fürsorge für die Verfolgten. Zunächst reduzierte das Social Insurance Committee wunschgemäß die Reichweite des Entwurfs ${ }^{44}$. Doch wurde dies alsbald durch eine andere Entwicklung überholt: Der Koordinierungsausschuß - zu dieser Zeit das maßgebliche Kontrollrats-Gremium - stimmte im März den gleichfalls im Manpower Directorate entworfenen Grundzügen über die Neuregelung des Systems der deutschen Sozialversicherung $\mathrm{zu}^{45}$, in die auch die Substanz des eigenständigen Entwurfs über die Stellung der Verfolgten in der Sozialversicherung integriert worden $w^{4{ }^{46}}$. Damit fiel die weitere Geschichte des Entwurfes über die Fürsorge für Verfolgte des Nationalsozialismus mit dem Schicksal des großangelegten alliierten Versuchs zur Reform der deutschen Sozialversicherung zusammen. Der Plan war, eine Einheitsversicherung zu schaffen, in der Kranken-, Renten- und Unfallversicherung organisatorisch zusammengefaßt werden sollten ${ }^{47}$.

Im Manpower Directorate wurden die Grundzüge zur Neugestaltung der deutschen Sozialversicherung zu einem recht umfangreichen Entwurf ausgearbeitet ${ }^{48}$. Darin waren zwar auch noch spezielle Bestimmungen zugunsten der durch den Nationalsozialismus Geschädigten vorhanden, die allerdings gemessen an den ursprünglichen Absichten des Social Insurance Committee erheblich bescheidener ausfielen. Nunmehr sollten nur noch solche Verfolgte, die einen Versicherungsanspruch erworben hatten, im Rahmen des Sozialversicherungssystems unterstützt werden, wenngleich zu 50 Prozent höheren Sätzen ${ }^{49}$. Mit einem Wort: Aus der Verfolgteneigenschaft resultierte jetzt nicht mehr automatisch ein Unterstützungsanspruch ${ }^{50}$.

Im Verlaufe der alliierten Diskussion um die Einbeziehung der Verfolgten in die Sozialversicherung gab es auch ausführliche Diskussionen um die Definition der „Opfer des Nazismus“, an denen sich neben dem Manpower Directorate eine ganze Reihe

42 ACA, Directorate of Manpower, Committee on Social Insurance, „Basic Principles of Social Insurance for Nazi Victims“, DMAN/P (46)14, 24.1. 1946, IfZ-Archiv, MF 260, OMGUS-ACA 2/101-3/6.

${ }^{43}$ Report from the Committee on Social Insurance including Unemployment Relief, DMAN/P (46)14 rev., 25. 2. 1946, IfZ-Archiv, MF 260, OMGUS 2/126-1/7-14.

44 "General Principles of Assistance to the Victims of Nazism", DMAN/P(46) 14 rev., 25.2. 1946, IfZ-Archiv, MF 260, OMGUS 2/126-1/7-14.

${ }^{45}$ Henry H. Ford (ACA/Manpower Directorate) an Finance Directorate, 24.4. 1946, IfZ-Archiv, MF 260, OMGUS 2/101-3/6.

46 ACA, Manpower Directorate, „Basic Principles of Social Insurance for Workers and Employees in Germany“, DMAN/P/46/11 rev., 20.3. 1946, IfZ-Archiv, MF 260, OMGUS 2/126-1/7-14.

47 Vgl. dazu Hans Günter Hockerts, Sozialpolitische Entscheidungen im Nachkriegsdeutschland. Alliierte und deutsche Sozialversicherungspolitik 1945 bis 1957, Stuttgart 1980, S. 26.

48 ACA, Manpower Directorate, "Proposed Compulsory Social Insurance Law in Germany“, DMAN/P(46) 105, Second rev., 30.8. 1946, IfZ-Archiv, MF 260, OMGUS 2/126-1/7-14.

49 Das entspricht der späteren Regelung in der DDR. Vgl. Deutsches Institut für Zeitgeschichte (Hrsg.), Handbuch der Deutschen Demokratischen Republik, o.O. 1964, S.639.

so Art. 73, 74, Proposed Compulsory Social Insurance Law (Anm. 48). 
anderer interessierter Direktorien beteiligten. Dabei verständigten sich die Alliierten darauf, daß nur die am härtesten betroffenen Verfolgten einbezogen werden sollten ${ }^{51}$. Der alliierte Entwurf über die Neuregelung der deutschen Sozialversicherung definierte als „Opfer des Nationalsozialismus“ schließlich nur diejenigen Personen, die vom NSRegime wegen ihrer politischen Überzeugungen, religiösen Anschauungen, Nationalität, Rasse oder wegen regimefeindlicher Aktivitäten in ein Gefängnis oder ein Konzentrationslager gesteckt worden waren ${ }^{52}$.

Nachdem die Fürsorgeansprüche der Verfolgten in den breiteren Kontext der Reform der deutschen Sozialversicherung gestellt waren, gerieten sie also offensichtlich unter den Druck, der aus dem alliierten Wunsch nach Kostendämpfung resultierte. Doch zuletzt wurde auch dieser Entwurf Makulatur und damit auch die vorgesehenen Bestimmungen zugunsten der Verfolgten bedeutungslos. Anfang 1948 ließ der Kontrollrat das Projekt fallen; die Vereinigten Staaten sahen sich mittlerweile mit massiven deutschen Widerständen gegen das Vorhaben konfrontiert und verzichteten darauf, eine gesamtalliierte Einigung auf diesem Feld zu versuchen ${ }^{53}$.

Faßt man die Bemühungen des Alliierten Kontrollrats um Fürsorgemaßnahmen zugunsten der Opfer des Nationalsozialismus zusammen, so wird deutlich, daß über die bereits während des Krieges in der Londoner European Advisory Commission getroffenen Abmachungen hinaus kaum weitere substantielle Einigungen erzielt wurden. Die damit verbundenen Fragen griffen zu tief in die Details der gesellschaftlichen Entwicklung in den vier Zonen Deutschlands ein, als daß es gelungen wäre, hier unter den Bedingungen der zunehmenden politischen Polarisierung eine gemeinsame Linie festzulegen. Bereits in einem frühen Stadium der alliierten Okkupation kam es so zu einem getrennten Vorgehen der Besatzungsmächte, ähnlich wie auf anderen Politikfeldern. Die Entwicklung der Fürsorge für die Verfolgten sowie vor allem auch der weiterführenden Maßnahmen verlief deshalb überwiegend auf zonaler Ebene. Den in der USZone entwickelten Strukturen kam im Hinblick auf die Gesamtentwicklung eine besondere Bedeutung zu, die es rechtfertigt, sie in den Mittelpunkt der Analyse zu rücken.

\section{Die Rolle der amerikanischen Militärregierung}

Die amerikanische Militärregierung in Deutschland (OMGUS) näherte sich dem Problem der Behandlung der Opfer des Nationalsozialismus zunächst unter dem praktischen Gesichtspunkt der Aufrechterhaltung der öffentlichen Ordnung. Zu diesem Zweck mußten dringend Fürsorgemaßnahmen für diejenigen Verfolgten getroffen werden, die in bitterer Not standen. Dazu gehörten insbesondere die zahlreichen aus den Konzentrationslagern befreiten Häftlinge. Neben den Juden stand diese besonders hart betroffene Gruppe bei den Fürsorgemaßnahmen zunächst ausdrücklich im Mittelpunkt. Dementsprechend wurde auch General Eisenhower als der Oberbefehlshaber der US-Besatzungstruppen in der Direktive JCS 1067 vom April 1945 angewiesen, in seiner Zone „alle diejenigen Personen, die auf Grund ihrer Rassezugehörigkeit, Nationalität, ihres Glaubensbekenntnisses oder ihrer politischen Ansichten gefangen oder in

\footnotetext{
51 Einen Überblick über den komplizierten interalliierten Definitionsprozeß findet sich in R. Peter Straus (ACA/Manpower Dir.), Summary of Action to Date on Definition of „Victims of Fascism“, Sommer 1946, IfZ-Archiv, MF 260, OMGUS-Manpower Dir. 7/46-1/11.

52 Proposed Compulsory Insurance Law, 20.9. 1946 (Anm. 48).

53 Vgl. Hockerts, Sozialpolitische Entscheidungen im Nachkriegsdeutschland, S. 51-85, v.a. S. 85.
} 
Gewahrsam gehalten worden waren, frei(zu)lassen und als verschleppte Personen

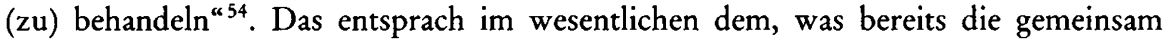
von den Alliierten formulierte Kapitulationsurkunde gefordert hatte. Somit standen zunächst sowohl die ausländischen als auch die deutschen Verfolgten unter dem besonderen Schutz der amerikanischen Militärregierung, beide besaßen Anspruch auf Betreuung als Displaced Persons.

Als eine besondere Gruppe der Displaced Persons wurden die "Persecutees“ auch in den amerikanischen Military Government Regulations definiert. Ein Verfolgter war demzufolge eine Person, deren uneingeschränkte Loyalität zur alliierten Sache feststand und die überdies beweisen könne, daß sie „(1) Jude ist; oder (2) wegen ihrer Farbe, Rasse, Religion, politischen Überzeugung oder Aktivitäten zugunsten der Vereinten Nationen, nicht aber wegen eines zivilen oder militärischen Verbrechens, in ein Gefängnis oder Konzentrationslager gesperrt wurde; oder (3) ein enger Familienangehöriger" solcher Personen sei ${ }^{55}$. Auch hier wurde also bereits Wert auf die Unterscheidung zwischen Inhaftierung aus Gründen NS-spezifischer Verfolgungsmaßnahmen und wegen "normaler" Vergehen gelegt.

Darüber hinaus wurden Deutsche und Ausländer im Rahmen der Fürsorge strikt getrennt. Die Betreuung der ausländischen und staatenlosen Verfolgten übernahmen Organisationen der UN oder private, insbesondere jüdische, Hilfsorganisationen. Die Fürsorge für die deutschen Verfolgten übertrug man demgegenüber, abgesehen von der ersten Betreuung nach der Befreiung aus einem KZ, deutschen Stellen. Die Military Government Regulations wiesen den öffentlichen Wohlfahrtsämtern die Aufgabe zu, Sorge zu tragen, daß deutsche Verfolgte mit DP's gleichgestellt wür$\operatorname{den}^{56}$. Auch auf deutscher Seite befürwortete man ausdrücklich, selbst nur deutsche Verfolgte zu betreuen ${ }^{57}$. Während also die Amerikaner aus verständlichen Gründen den Deutschen keine Verfügungsgewalt über die ehemaligen ausländischen Verfolgten, sei es auch nur im Rahmen der Fürsorge, einräumen wollten, lehnten umgekehrt die deutschen Stellen ihrerseits die Verantwortung für diesen Personenkreis ab.

Allerdings war in den Military Government Regulations auch festgelegt, daß die Autorität zur Bestimmung des Persecutee-Status ausschließlich bei der Militärregierung liege und insbesondere deutsche Behörden nicht dazu berechtigt seien ${ }^{58}$. Die Gewährung dieses Status war mit dem Anspruch auf Unterstützung durch die United Nations Relief and Rehabilitation Administration (UNRRA) bzw. später die International Refugee Organization (IRO) verbunden. Dieses Vorrecht sollte den Deutschen aus naheliegenden Gründen nicht zuerkannt werden. Aus praktischen Erwägungen war die Militärregierung aber gezwungen, den Deutschen zumindest die Auswahl der unter deutsche Betreuung fallenden Personen zu überlassen. Die Definition in den Military Government Regulations bildete freilich für die deut-

54 JCS 1067, Abs. 13, in: Um den Frieden mit Deutschland, S. 58-73, S. 64.

55 USFET, Military Government Regulations, 20.-101.3, hier Ausgabe v. 1.12. 1947, IfZ-Archiv, Dk 133.001(a). Vgl. auch Guide to the Care of Displaced Persons in Germany, SHAEF, G-5 Division, May 1945, IfZArchiv, Dk 090.005.

${ }^{56}$ USFET, vermutl. 10.8. 1945, Title 7, Public Welfare, Section 7-255, hier zitiert nach William S. Fitzer an Direktor Buchmann, Stuttgart, 23.5. 1946, IfZ-Archiv, MF 260, OMGUS-WB 12/26-2/26.

57 Ebenda; Charles E. Stewart an Director, OMGUS, 21. 8. 1946, IfZ-Archiv, MF 260, OMGUS-CAD 5/323-3/ 5.

58 Military Government Regulations (Anm. 55). 
schen Definitionen eine Richtschnur bei der Bestimmung des Verfolgtenstatus und für die amerikanische Militärregierung eine ständige Handhabe zum Eingreifen.

Bei den deutschen Fürsorge-Bemühungen wirkte die amerikanische Militärregierung überwachend, teilweise aber auch durch eigene Initiativen mit. Besondere Aufmerksamkeit der US-Militärregierung galt dabei den verbliebenen kleinen jüdischen Restgemeinden, die außerhalb des Systems der direkten Betreuung durch die Militärregierung oder der UNRRA standen und die auch in weit geringerem Maße als die Juden in DPLagern auf die Unterstützung der internationalen jüdischen Hilfsorganisationen zählen konnten. Dabei formulierten die Amerikaner in der ersten Zeit der Besatzung klare Vorgaben für die deutschen Stellen. Hierzu gehörte die Zuteilung erhöhter Lebensmittelrationen ${ }^{59}$, die bevorzugte Beschaffung von Wohnraum und Wohnungseinrichtungen $^{60}$ sowie von Arbeitsplätzen ${ }^{61}$, aber auch bevorzugte Behandlung in der Sozialversicherung $^{62}$ und bei der Vergabe von Gewerbelizenzen ${ }^{63}$. Diese Vorgaben setzten die Militärregierungen der einzelnen Länder der US-Zone unter anderem durch Anweisungen an die jeweiligen Landesregierungen um.

Am Beispiel der durch die amerikanische Militärregierung im Oktober 1945 verfügten erhöhten Lebensmittelration für die rassisch, religiös und politisch Verfolgten in der US-Zone ${ }^{64}$ läßt sich die geplante Reichweite dieser Fürsorgemaßnahmen verdeutlichen. Im Sommer 1948 wurde diese Regelung mit der Begründung abgeschafft, die allgemeine Ernährungssituation habe sich erheblich gebessert und die gesellschaftliche und wirtschaftliche Eingliederung der Verfolgten des Nationalsozialismus erfordere ihre Gleichstellung mit der übrigen Bevölkerung ${ }^{65}$. Die Bemühungen der amerikanischen Militärregierung um die Fürsorge für diese Gruppe in ihrer Besatzungszone waren streng begrenzt auf eine übergangsweise Unterstützung mit dem Ziel, die ehemaligen Verfolgten physisch und sozial wieder soweit zu rehabilitieren, daß sie am normalen Leben teilnehmen konnten. Jeder Tendenz zur festen Etablierung der Hilfsmaßnahmen trat OMGUS von Anfang an entgegen. Spätestens nach dem Erlaß von Wiedergutmachungsgesetzen sollten diese ein Ende finden, was an akuten Problemen blieb, der allgemeinen Fürsorge anheimfallen. Hauptsorge war dabei die Befürchtung, daß sich die Verfolgten infolge der Fürsorgemaßnahmen als gesellschaftliche Gruppe dauerhaft verfestigen könnten. General Lucius D. Clay erklärte deshalb 1948, „die endgültige Lösung für diese verfolgten Gruppen ist die Umsiedlung oder die Integration dieser Menschen in die deutsche Wirtschaft auf gleicher Grundlage wie die anderen Bürger" ${ }^{\text {666 }}$. Die amerikanische Militärregierung dachte keinesfalls daran, die Verfolgten des Nationalsozialismus aufgrund ihres Schicksals gesellschaftlich oder politisch herauszuheben. Vielmehr entsprach es ihrem Gesamtinteresse, diese, sofern sie nicht auswanderten, möglichst nahtlos in die entstehende deutsche Nachkriegsgesellschaft einzugliedern.

59 Ebenda, Title 12, Food and Agriculture, Part 3-Sec C. para. 320, „Ration Scales for Persons Persecuted by Nazi Regime".

60 Ebenda, Title 15, Manpower, Part 4 - Sec.C - para. 435, „Priorities in Allocation of Housing Space“.

61 Ebenda, Title 15, Manpower, Part 3, Sec. B - para. 315 (a), „Preferential Treatment for Those Discriminated Against by Nazis".

62 Ebenda, Title 15, Manpower, Part 7 - Sec A. para. 700.5, „Priority Treatment for Nazi Victims“; ebenda, para. 700.6, „Special Payments for Disability Inflicted by Nazis“.

63 Ebenda, para 700.7, „Priorities in Issuance of Trade Permits“.

64 Siehe dazu auch etwa H.H. Newman to Commanding Generals, Special Ration in the U.S. Zone of Germany for Persons Persecuted by the Nazi Regime, 15.10. 1945, IfZ-Archiv, MF 260, OMGUS-CAD 5/323-3/6.

${ }^{65}$ Bipartite Control Office, BICO/P (48) 206, 4.8. 1948, IfZ-Archiv, MF 260, OMGUS 8/66-2/2.

${ }^{66}$ Clay an Murray D. van Wagoner, ca. August 1948, IfZ-Archiv, MF 260, OMGUS-CAD 3/173-1/19. 


\section{Fürsorge für Verfolgte des Nationalsozialismus in Süddeutschland}

\section{Verfolgtenbetreuung zwischen Selbsthilfe und staatlicher Unterstützung}

a) Bayern

Betreuung und Fürsorge für die Opfer des Nationalsozialismus vollzogen sich in der US-Zone unter den Rahmenbedingungen, die die Militärregierung setzte. Dennoch ergaben sich neben manchen gemeinsamen Entwicklungstendenzen auch verschiedene spezielle Entwicklungen in den einzelnen Ländern. So fanden sich unter allen Ländern der amerikanischen Zone in Bayern mit weitem Abstand die meisten ehemaligen KZHäftlinge, unter anderem auch deshalb, weil kurz vor Kriegsende viele Häftlinge in das noch unbesetzte Bayern verbracht worden waren. Als die beiden großen bayerischen Lager Dachau und Flossenbürg im April 1945 befreit wurden, befanden sich dort noch etwa 32.000 bzw. 15.000 Häftlinge, weitere 50-60.000 Häftlinge wurden in Außenlagern oder auf Evakuierungsmärschen befreit ${ }^{67}$.

Nach dem Zusammenbruch entstanden teils aus eigener Initiative der Verfolgten, teils durch Vermittlung des Bayerischen Roten Kreuzes sowie kommunaler oder Landkreisbehörden in vielen Orten Bayerns KZ-Betreuungsstellen. Diese kümmerten sich um die Unterstützung der befreiten Häftlinge, soweit diese nicht als UN-Ausländer Anspruch auf Hilfe im Rahmen der DP-Betreuung besaßen ${ }^{68}$. Eine Abteilung für politische Verfolgte des Roten Kreuzes mußte im Juni 1946 auf Anordnung der Militärregierung aufgelöst werden ${ }^{69}$. Dagegen half die Süddeutsche Ärzte- und Sanitätshilfe der Centrale Sanitaire Suisse bei der medizinischen Betreuung der ehemaligen KZ-Häftlinge. Hier hatten sich ehemalige Emigranten und Häftlinge vornehmlich des linken politischen Spektrums zusammengeschlossen, die in Bayern, aber auch in WürttembergBaden und Großhessen KZ-Heimkehrern ärztliche Pflege zuteil werden ließen und sie mit Medikamenten versorgten ${ }^{70}$. Da die rassisch Verfolgten Wert auf eine eigene Betreuung legten, wurde im Auftrag der amerikanischen Militärregierung am 15. Mai 1945 zunächst in München das Hilfswerk für die von den Nürnberger Gesetzen Betroffenen gegründet. Ende 1945 wurde es als Bayerisches Hilfswerk landesweit zugelassen. Es kümmerte sich um „Volljuden“, Zigeuner und auch um rassisch verfolgte Menschen aus

67 Vgl. dazu Günther Kimmel, Das Konzentrationlager Dachau. Eine Studie zu den nationalsozialistischen Gewaltverbrechen, in: Bayern in der NS-Zeit, hrsg. v. Martin Broszat und Elke Fröhlich, Bd. II: Herrschaft und Gesellschaft im Konflikt, München 1979, S.410; Toni Siegert, Das Konzentrationslager Flossenbürg. Ein Lager für sogenannte Asoziale und Kriminelle, in: ebenda, S. 483-485; Barbara Distel, Der 29. April 1945. Die Befreiung des Konzentrationslagers Dachau, in: Dachauer Hefte 1 (1985), S.3-11; Juliane Wetzel, „Mir szeinen doh“. München und Umgebung als Zuflucht von Überlebenden des Holocaust 1945-1948, in: Von Stalingrad zur Währungsreform. Zur Sozialgeschichte des Umbruchs in Deutschland, hrsg. v. Martin Broszat, Klaus-Dietmar Henke u. Hans Woller, München 1988, S. 337.

${ }^{68}$ Siehe dazu Dr. Walter an OMGB-Political Affairs, ICD, 6. 8. 1946, IfZ-Archiv, MF 260, OMGUS 10/109-2/ 2.

69 Information from Otto Aster to Peter G. Harnden (OMGB/ICD), 3. 8. 1946, IfZ-Archiv, MF 260, OMGUS 10/109-2/1. Auch die Übernahme der praktischen Durchführung der Flüchtlingsbetreuung durch das Rote Kreuz scheiterte am Veto der Militärregierung. Vgl. Franz J. Bauer, Flüchtlinge und Flüchtlingspolitik in Bayern 1945-1950, Stuttgart 1982, S. 34-39.

70 Siehe Mitteilungsblatt des Landesausschusses der politisch Verfolgten, Nr. 1, 1. 12. 1946, "Die gesundheitliche Betreuung der politisch Verfolgten“; Public Welfare Branch, OMGB, 22.12. 1945, Memorandum betr. Süddeutsche Ärzte- und Sanitätshilfe, IfZ-Archiv, MF 260, OMGUS 12/26-2/26. 
jüdisch-christlichen Ehen sowie um sogenannte „Nicht-Glaubensjuden“71. Die jüdischen DP's, $d$. h. die nichtdeutschen Juden, gründeten hingegen bereits 1945 eine eigene Organisation, das Zentralkomitee der befreiten Juden in Bayern, das sich 1946 auf die ganze US-Zone ausdehnte ${ }^{72}$.

Damit hatte sich also von Anfang an eine deutliche Gruppenbildung unter den ehemaligen Verfolgten des Nationalsozialismus vollzogen: Die politischen und in geringerer Zahl auch die religiös verfolgten Deutschen wurden durch die KZ-Betreuungsstellen unterstützt. Deutsche rassisch Verfolgte, also in erster Linie überlebende Juden sowie Sinti und Roma, erhielten Beistand durch das Bayerische Hilfswerk. Bei ausländischen Verfolgten wurde unterschieden zwischen Verfolgten, die in Lagern lebten und UNRRA-Hilfe erhielten, und Verfolgten außerhalb der Lager, für die sich - sofern es sich dabei um bekennende Juden handelte - in erster Linie das Zentralkomitee der befreiten Juden einsetzte, das die Unterstützung internationaler jüdischer Hilfsorganisationen wie dem American Joint Distribution Committee und der Jewish Agency besa $\AA^{73}$. Bei den Betreuungsstellen waren Ende 1945 ca. 10-11.000 Menschen als politisch Verfolgte anerkannt, bis Frühjahr 1947 stieg diese Zahl auf etwa 18.000 ${ }^{74}$. Die vom Bayerischen Hilfswerk betreute Gruppe der deutschen Juden sowie der Zigeuner war bis Anfang 1947 gleichfalls auf etwa 18.000 Menschen angewachsen ${ }^{75}$. Das Zentralkomitee betreute Anfang 1947 etwa 29.000 jüdische DP's, die außerhalb der UNRRABetreuung in Bayern lebten. Bis zur Währungsreform war für diese Gruppe die Unterstützung durch ausländische jüdische Hilfsorganisationen entscheidend, erst danach nahmen sie zunehmend die Unterstützung deutscher Stellen in Anspruch ${ }^{76}$. Insgesamt wurden also in Bayern bis Anfang 1947 ca. 65.000 Menschen als Verfolgte des Nationalsozialismus betreut.

Am 31. Oktober 1945 machte Militärgouverneur Walter J. Muller den bayerischen Ministerpräsidenten Wilhelm Hoegner mit der in den amerikanischen Bestimmungen festgelegten Verpflichtung der deutschen Behörden zur Betreuung deutscher Verfolgter bekannt. Er wies ihn ausdrücklich an, allen Fürsorgestellen „den Befehl zu geben, daß Juden, politisch Internierte und deutsche Insassen von Konzentrationslagern, die in derartige Lagern aus rassischen oder religiösen Gründen oder wegen Widerstandes gegen die Nazis kamen, bevorzugt behandelt und bevorzugt unterstützt " würden. Es sei sicherzustellen, daß solche Personen angemessen wohnen, mit Möbeln, guter Kleidung und Brennmaterial versorgt würden sowie umfassende medizinische Betreuung erhielten. Es müsse alles dafür getan werden, „um diese Leute finanziell unabhängig zu

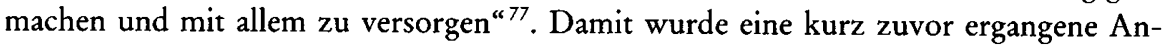

71 Vgl. Juliane Wetzel, Jüdisches Leben in München 1945-1951. Durchgangsstation oder Wiederaufbau?, München 1987, S. $37 \mathrm{ff}$. Siche auch Louis Miniclier an Land Director, OMGB, 6.2. 1948, „Minority Groups in Bavaria - Jews“, IfZ-Archiv, MF 260, OMGUS 13/141-1/1.

72 Wetzel, Jüdisches Leben in München, S. 144ff.; Miniclier an Land Director, 6. 2. 1948 (Anm. 71).

73 Vgl. Kurt R. Großmann, Die jüdischen Auslandsorganisationen und ihre Arbeit in Deutschland, in: Die Juden in Deutschland. Fin Almanach 1951/52, hrsg. v. Heinz Ganther, Frankfurt a.M. u. München 1953, S. 91-136.

74 Otto Aster, Bericht über die Betreuung der politisch Verfolgten, 21. 10. 1946, IfZ-Archiv, NL Hoegner, ED 120, Bd. 116; Rechenschaftsbericht des Staatskommissars für rassisch, religiös und politisch Verfolgte, 15.9. 1946-15. 5. 1947, If́Z-Archiv, MF 260, OMGUS 13/141-1/1.

75 Rechenschaftsbericht des Staatskommissars (Anm. 74).

76 Aussage v. Blessin, Untersuchungsausschuß zur Prüfung der Vorgänge im Landesentschädigungsamt (UA. LEA), 7. Sitzung, 14.9. 1951, S. 46, BayLt-Archiv.

77 Walter J. Muller an Hoegner, 31. 10. 1945, Archiv des Bayerischen Landesentschädigungsamts. 
weisung der Public Welfare Branch, die sich nur auf die bevorzugte Betreuung der Juden bezogen hatte, auch auf andere Verfolgte ausgeweitet ${ }^{78}$.

Die Public Welfare Branch hatte Hoegner dazu veranlaßt, am 26. Oktober 1945 ein Staatskommissariat für rassisch Verfolgte zu gründen, das dem Innenministerium unterstand. Der für das Amt des Staatskommissars ausgewählte Hermann Aumer sollte in Zusammenarbeit mit dem Ministerpräsidenten sowie mit den zuständigen Ministerien und Landesbehörden den rassisch verfolgten bayerischen Juden alle erforderliche Hilfe zukommen lassen. In seiner Ernennungsurkunde war u. a. der Auftrag enthalten, Sorge dafür zu tragen, „daß der jüdische Bevölkerungsteil des Landes Bayern wieder ein gesunder Faktor in der bayerischen Wirtschaft wird"79. Leider dachte Aumer hauptsächlich an seine eigene wirtschaftliche Gesundung, und so ordnete die amerikanische Militärregierung im August 1946 an, ihn wegen Unterschlagungen zu entlassen und ihn von allen öffentlichen Ämtern fernzuhalten ${ }^{80}$.

Der Weg der Einrichtung von Staatskommissariaten wurde zu dieser Zeit in Bayern mehrfach beschritten. So wurde neben dem Staatskommissar für die Betreuung der Juden auch ein Staatskommissar für Flüchtlingsfragen bestimmt ${ }^{81}$. Dahinter steckte die Absicht, nur temporäre Sonderbehörden zu schaffen, die sich nach der Beendigung ihrer Aufgaben leichter auflösen ließen als in die traditionelle Verwaltung integrierte Behörden. Innenminister Josef Seifried erläuterte später, „alle diese Funktionen, die sich aus der Katastrophe ergeben haben, wie die Betreuung der Flüchtlinge und der rassisch, religiös und politisch Verfolgten, (sci. sollten) nur in der Art eines Staatskommissariats wahrgenommen werden, um später wieder zu verschwinden" ${ }^{82}$.

Auch die politisch Verfolgten forderten nun energisch ein Staatskommissariat für ihre Belange ${ }^{83}$, worauf schließlich am 26. März 1946 Otto Aster, ein Kommunist und ehemaliger Metzger, mit der Leitung eines solchen Amtes beauftragt wurde. Seine vordringlichen Aufgaben waren, den Personenkreis der politisch Verfolgten zu bestimmen, Hilfsmaßnahmen zur Behebung dringender Notmaßnahmen durchzuführen sowie Wiederaufbaumöglichkeiten für die politischen Verfolgten zu schaffen. In den Land- und Stadtkreisen sollten Betreuungsstellen errichtet werden, in die die bisher bestehenden KZ-Betreuungsstellen nach Möglichkeit zu übernehmen waren ${ }^{84}$.

Die beiden Staatskommissare mußten zunächst mit recht bescheidenen Mitteln auskommen. Soweit ihre Kräfte reichten, halfen sie ihren Schützlingen durch die Ausgabe von Schwerarbeiterzulagen auf ihre Lebensmittelkarten, die Beschaffung von Hausrat, Kleidern, Wohnungen, Arbeit oder auch Krankenbehandlungen. Der Staatskommissar für die Betreuung der Juden zahlte Anfang 1946 einen Teil der Judenvermögensabgaben zurück, zudem konnte er einer Anzahl von Juden mit Darlehen zur Geschäftsgründung verhelfen ${ }^{85}$. Doch bei all diesen Hilfsmaßnahmen machte sich der allgemeine Mangel stark bemerkbar, stets konnte nur ein Bruchteil des Benötigten bereitgestellt werden.

${ }^{78}$ Sitzung des bayerischen Ministerrats am 24. 10. 1945, IfZ-Archiv, NL Hoegner, ED 120, Bd. 354.

79 Bestallungsurkunde für Hermann Aumer, 26.10. 1945, BayHStA, MA 114262.

${ }^{80}$ Paul W. Friedmann, The Philipp Auerbach Tragedy, 17.12. 1952, YIVO-Archiv, RG 347, AJC Records, GEN-10, Box 36.

$81 \mathrm{Vgl}$. Bauer, Flüchtlinge und Flüchtlingspolitik, S. $42 \mathrm{ff}$.

82 Aussage v. Seifried, 6. UA.LEA, 31.8. 1951, S. 105, BayLt-Archiv.

${ }^{83}$ Sitzung des bayerischen Ministerrats am 7.1. 1946, IfZ-Archiv, NL Hoegner, ED 120, Bd. 356.

${ }^{84}$ Rundschreiben Nr.1156a 30 des bayerischen Innenministeriums an die Regierungspräsidenten und den Staatskommissar für die politisch Verfolgten, 4.5. 1946, BayHStA, MA 114263.

${ }^{85}$ Vormerkung Walter Roemer, 18.5. 1946, BayMJ, 1101a, H. 1. 
Im Sommer 1946 forderte die Militärregierung die bayerische Regierung zur Vereinigung der Staatskommissariate sowie zur Eingliederung ihrer Aufgaben in die Wohlfahrtsabteilung des Innenministeriums auf. Sie befürchtete insbesondere, die Existenz von selbständigen Staatskommissariaten zur Betreuung der Opfer des Nationalsozialismus könne auf die Dauer dazu führen, daß sich die Verfolgten als gesellschaftliche Sondergruppe etablierten ${ }^{86}$. Die bayerische Regierung beschloß daraufhin zwar die Zusammenlegung der bisherigen Stellen in ein gemeinsames „Staatskommissariat für die Opfer des Faschismus“, doch die von der Militärregierung intendierte Eingliederung in die Wohlfahrtsabteilung des bayerischen Innenministeriums unterblieb. Deshalb legte auch die Militärregierung Wert darauf, daß diese Dienststelle aufgelöst werden sollte, sobald es die Verhältnisse zuließen ${ }^{87}$.

Nach der Zusammenlegung der beiden Staatskommissariate suchte die bayerische Regierung nach einem geeigneten Leiter. Die Wahl fiel auf den prominenten jüdischen Funktionär Philipp Auerbach, für den sich mehrere jüdische Interessenvertretungen eingesetzt hatten ${ }^{88}$. Auerbach stammte aus einer angesehenen Hamburger jüdischen Familie und hatte sich als Chemiker und Kaufmann betätigt. Während des Krieges verbrachte er mehrere Jahre in deutschen Gefängnissen und Konzentrationslagern. Anschließend war er bei der Regierung der Nordrhein-Provinz für die Betreuung ehemaliger Verfolgter und Flüchtlinge zuständig. Als Auerbach auf eigene Faust eine nationalsozialistische Belastung des damaligen Oberpräsidenten der Nordrhein-Provinz und späteren Bundesinnenministers Robert Lehr nachweisen wollte, ordnete die britische Militärregierung seine Entlassung an. Seither konzentrierte er sich auf seine Tätigkeit als Vorsitzender des Landesverbandes der jüdischen Kultusgemeinden der britischen Zone ${ }^{89}$.

Auerbach trat seine Stelle am 15. September 1946 an, und am 10. Oktober ernannte ihn die Staatsregierung förmlich zum Staatskommissar für die Opfer des Faschismus. Erneut unterstrich der Ministerrat den temporären Charakter dieses Amtes und sah deshalb bewußt von der Verbeamtung Auerbachs ab ${ }^{90}$. Bereits nach kurzer Zeit mußte auf Anordnung der Militärregierung die Amtsbezeichnung in „Staatskommissariat für die rassisch, religiös und politisch Verfolgten" geändert werden" ${ }^{91}$. Damit reagierte sie auf die mittlerweile erfolgten Gründungen von politischen Vereinigungen der Verfolgten, die unter dem Begriff „Opfer des Faschismus“ firmierten und ihn damit politisch besetzt hatten ${ }^{92}$.

Spätestens seit 1947 war dieses Amt die zentrale Instanz bei der Verfolgtenbetreuung

${ }^{86}$ Siehe etwa Aktennotiz v. Herwarths über Besprechung bei Albert C. Schweitzer (Leiter der OMGB-Civil Administration Division), 16. 7. 1946, BayHStA, MA 114263.

87 Schweitzer an Hoegner, 14. 11. 1946, IfZ-Archiv, ED 120, NL Hoegner, Bd. 112.

88 Telegramm der Interessenvertretung jüdischer Gemeinden und Kultusvereinigungen der drei Zonen an Ministerpräsident Hoegner v. 20. 8. 1946, Landgericht München I, Az. 2 KLs 1/52, Bd. IV/4; Central Committee of Liberated Jews in the American Occupied Zone in Germany an Innenminister Seifried, 30. 8. 1946, ebenda. Vgl. auch Constantin Goschler, Der Fall Philipp Auerbach. Wiedergutmachung in Bayern, in: Wiedergutmachung in der Bundesrepublik Deutschland, hrsg. v. Ludolf Herbst u. Constantin Goschler, München 1989, S. 79.

${ }^{89} \mathrm{Vgl}$. Urteil in der Sache Auerbach und drei Andere, 14. 8. 1952, Landgericht München I, Az. 2 KLs 1/52.

90 Protokoll der Sitzung des bayerischen Ministerrats am 5.9. 1946, BayStK-Archiv.

91 Robert A. Reese (OMGB/Internal Affairs Division) an Ministerpräsident Hans Ehard am 3.1. 1947, BayHStA, MA 114263

92 ACA, Directorate of Prisoner of War and Displaced Persons, Definition of the Terms "Victims of Fascism" and "Victims of Nazism“, 15.7. 1946, IfZ-Archiv, MF 260, OMGUS-ACA 2/101-3/6. 
in Bayern. In jedem Regierungsbezirk besaß der Staatskommissar einen Bevollmächtigten, der die Verbindung zu den einzelnen Betreuungsstellen herstellte. Neben diesem analog zum traditionellen dreigliedrigen bayerischen Verwaltungssystem aufgebauten staatlichen Apparat bestand seit Ende 1946 ein Landesausschuß der politisch Verfolgten, der eine beratende Funktion beim Staatskommissariat innehatte und so die Interessen der Verfolgten vertreten sollte. Die Gründung des Landesauschusses war auf Anforderung der Militärregierung erfolgt, die damit auf die zahlreichen Klagen aus den Reihen der Verfolgten reagierte. In ihm waren alle fünf im bayerischen Landtag vertretenen Parteien repräsentiert, die ihrerseits ehemalige Verfolgte aus ihren Reihen delegierten ${ }^{93}$.

In einem Rechenschaftsbericht vom Mai 1947 erläuterte Auerbach die Tätigkeit seines Staatskommissariats ${ }^{94}$. Es besaß nun die direkte Dienstaufsicht über das Bayerische Hilfswerk für die durch die Nürnberger Gesetze Betroffenen, die KZ-Betreuungsstellen und das Zentralkomitee der befreiten Juden in der US-Zone. Somit war das Staatskommissariat nicht nur für die deutschen rassisch, religiös und politisch Verfolgten, sondern auch für jüdische DP's, soweit sie nicht von der UNRRA betreut wurden, zuständig ${ }^{95}$. Neben der Gewährung von Renten und Krediten sorgte das Staatskommissariat für Sonderverteilungen von Textilien, Möbeln, Lebensmitteln und anderen Gebrauchsgegenständen, unterhielt eine Reihe von Erholungsheimen für die Verfolgten, setzte sich für die Errichtung und Pflege von Friedhöfen und Gedenkstätten ein, unterstützte Verfolgte beim Studium, verteilte Wohnungen und war an der beruflichen Eingliederung und Arbeitsvermittlung beteiligt. Daneben förderte es auch die Ausbildung auswanderungswilliger jüdischer DP's, um deren Startchancen in ihrer neuen Heimat zu verbessern. Schließlich bestanden auch eigene Abteilungen für die Wiedergutmachungsgesetzgebung sowie für Entnazifizierungsfragen ${ }^{96}$.

Schon bald nach Aufnahme seiner Tätigkeit in Bayern hatte Auerbach das Ziel verfolgt, „daß die rassisch, religiös und politisch Verfolgten vollkommen aus der Wohlfahrt ausscheiden ${ }^{497}$, und wurde darin auch durch die für ihn zuständige Wohlfahrtsabteilung des Innenministeriums bestärkt. Die Verfolgten sollten in Zukunft ausschließlich über das Staatskommissariat und die ihm untergeordneten Hilfsstellen unterstützt werden und nicht mehr durch die allgemeinen öffentlichen Wohlfahrtseinrichtungen. Schließlich setzte Auerbach durch, daß zum 1. Juli 1947 eine entsprechende Regelung in Kraft trat ${ }^{98}$. Indem die Verfolgten aus dem Rahmen der allgemeinen Fürsorge herausgenommen wurden, sollte die besondere Natur ihrer Ansprüche unterstrichen werden, der man durch ein Almosen nicht gerecht würde.

Dieser Kurs erzeugte aber auch Widerstände. Der von Auerbach zielstrebig verfolgte Aufbau einer Sonderverwaltung für die Verfolgten des Nationalsozialismus schuf nicht nur Mißmut in manchen bayerischen Kreisen, sondern lief auch den Interessen der

93 Vgl. Mitteilungsblatt des Landesausschusses der politisch Verfolgten, Nr.1, 1.12. 1946, „Bildung eines Landesausschusses der politisch Verfolgten".

94 Siehe zum folgenden Rechenschaftsbericht des Staatskommissars für rassisch, religiös und politisch Verfolgte, 15.9. 1946-15. 5. 1947, OMGB, IfZ-Archiv, MF 260, OMGUS 13/141-1/1.

95 Muller an Hoegner, 18. 10. 1946, BayHStA, MA 114263.

96 Bericht des Staatskommissars für rassisch, religiös und politisch Verfolgte für die Militärregierung von Bayern, Januar 1948, IfZ-Archiv, MF 260, OMGUS 13/141-1/1.

97 Auerbach an Eckmaier (Ministerialrat im bayer. Finanzministerium), 18. 12. 1946, BayHStA, MF 69409.

98 Memorandum Minicliers v. 31. 1. 1947, OMGB, IfZ-Archiv, MF 260, OMGUS 13/141-1/1; Hans P. Thomsen (Welfare Refugee Officer, Ansbach) an Public Welfare Branch, OMGB, 16. 8. 1947, ebenda, 15/102-1/31. 
amerikanischen Militärregierung entgegen. Bereits Mitte 1947 kam diese in einer Untersuchung zu der Auffassung, daß der bayerische Staatskommissar seine offizielle Position unter anderem dazu mißbrauche, die politischen und wirtschaftlichen Ziele einer Minderheitsgruppe, d. h. der Verfolgten, innerhalb der deutschen Wirtschaft zu begünstigen ${ }^{99}$. Das widersprach der amerikanischen Politik, wie sie bei einem Besuch des Assistant Secretary of War Peterson in der amerikanischen Besatzungszone nochmals ausdrücklich formuliert worden war: Es müsse verhindert werden, daß sich die Verfolgten infolge einer bevorzugten Behandlung als eine von der übrigen Gesellschaft getrennte Bevölkerungsgruppe auf Dauer etablierten und damit den Unmut der Bevölkerung auf sich zögen ${ }^{100}$. Deshalb vertrat auch die Public Welfare Branch der amerikanischen Militärregierung in Bayern die Auffassung, daß die bevorzugte Behandlung der Verfolgten spätestens mit dem Inkrafttreten der endgültigen Wiedergutmachungsgesetzgebung ein Ende finden müsse ${ }^{101}$. In der Abneigung, durch fortgesetzte Fürsorgemaßnahmen eine Problemgruppe zu verfestigen, trat die für alle ihre Aktivitäten im Bereich der Fürsorge für die ehemaligen Verfolgten, aber auch etwa der Flüchtlinge und Vertriebenen, typische Maxime der amerikanischen Besatzungspolitik zutage ${ }^{102}$.

\section{b) Württemberg-Baden}

Auch in Württemberg-Baden gingen die ersten Fürsorgemaßnahmen zugunsten deutscher Opfer des Nationalsozialismus vorrangig von Initiativen der ehemaligen Verfolgten in Zusammenarbeit mit kommunalen Wohlfahrtseinrichtungen aus. So gründeten etwa in Stuttgart die ersten Heimkehrer aus den Konzentrationslagern und Gefängnissen, die meisten davon langjährige politische Häftlinge, in Verbindung mit dem städtischen Wohlfahrtsamt eine Betreuungsstelle. Sie kümmerten sich zunächst darum, die Rückkehr befreiter Häftlinge in ihre Heimatstadt zu organisieren. Jeder Rückkehrer bekam neben der üblichen Wohlfahrtsunterstützung einen Betrag von 30 RM. Ein kleiner Teil erhielt zudem eine Ehrengabe der Stadt Stuttgart in Höhe von 200 bis 300 RM, manche auch einige hundert Reichsmark für Kleider und Hausratbeschaffung aus Wohlfahrtsmitteln ${ }^{103}$. Außerhalb Stuttgarts war die Lage für die heimkehrenden und notleidenden Verfolgten meist noch dürftiger, sofern nicht in vereinzelten Fällen Sammlungen für diese Leute veranstaltet wurden. Dabei kam es aber auch zu Konflikten mit der Militärregierung, insbesondere wenn dies zu unkontrollierten Selbsthilfemaßnahmen der Verfolgten führte ${ }^{104}$.

Staatlicherseits wurde dagegen über längere Zeit kaum etwas getan. Dies hatte auch damit zu tun, daß es im Unterschied zu Bayern im südwestdeutschen Raum nicht

99 Dossier von Charles E. Boyle (OMGUS/Prisoner of War \& Displaced Persons Division) an Political Affairs, 25.7. 1947, IfZ-Archiv, MF 260, OMGUS 3/169-2/158.

100 Memorandum Minicliers v. 4.6. 1947, IfZ-Archiv, MF 260, OMGUS 13/141-1/1.

101 Al D. Sims (OMGB/Internal Affairs Division) an OMGUS, Internal Affairs \& Communications Division, 8. 11. 1947, IfZ-Archiv, MF 260, OMGUS-CAD 5/323-3/5.

102 Siehe etwa die Ansprache Clays vor dem Plenum des Stuttgarter Länderrats am 4.2. 1947, in der er davor warnte, bei den Flüchtlingen ein neues Minderheitenproblem entstehen zu lassen, in: Akten zur Vorgeschichte der Bundesrepublik Deutschland 1945-1949, (AVBRD), Bd. 2, Januar-Juni 1947, hrsg. v. Bundesarchiv u. d. Institut für Zeitgeschichte, München u. Wien 1979, S. 186.

${ }^{103}$ Referat Karl Keims über die Aufgaben der Betreuungsstellen und Bericht über die Betreuung in Stuttgart, in: Protokoll der Landeskonferenz der politisch Verfolgten des Naziregimes Württemberg-Baden am 17. März 1946 in Stuttgart, VVN/BW-Archiv, Geschichte/Dokumente der VVN; Wortlaut der Unterstützungsbestimmungen für Verfolgte des Nationalsozialismus der Stadt Stuttgart in: Runderlaß des Innenministeriums Nr. IX 439, 17. 12. 1945, IfZ-Archiv, MF 260, OMGUS 12/26-2/26.

104 Siehe etwa das Beispiel Mannheim, Material dazu in IfZ-Archiv, MF 260, OMGUS-WB 12/26-2/26. 
gelungen war, unmittelbar an die Verwaltungstradition der ehemaligen Länder anzuknüpfen. Die Grenze zwischen der französischen und amerikanischen Besatzungszone durchschnitt auf einige Jahre hin das vorher verwaltungsmäßig, kulturell und wirtschaftlich zusammengehörige Gebiet Württembergs, außerdem gehörten zur US-Zone nun auch Teile des ehemaligen Baden ${ }^{105}$. Noch in der Zeit des kurzen französischen Interims wurde in Stuttgart ein „Amt für Wiedergutmachung “ eingerichtet, das aber anfangs nur im Bereich Groß-Stuttgart tätig war. Nach dem Wechsel der Besatzungsmacht und der Bestätigung einer württemberg-badischen Landesregierung am 14. September 1945 wurde es zunächst im Bereich der Landesverwaltung für Inneres weitergeführt. Aufgabe des Amts für Wiedergutmachung war die Registrierung und - im Falle dringender Not - Unterstützung der Verfolgten des Nationalsozialismus, soweit es die bescheidenen Mittel zuließen. Dabei stand ihm ein Beirat zur Seite, in dem neben einer Anzahl von Verfolgtenvertretern die Militärregierung, etliche Landkreise und andere mit der Verfolgtenbetreuung befaßte Stellen vertreten waren.

Jedoch war das Verhältnis zwischen dem noch von der französischen Militärregierung eingesetzten Amtsleiter Franz Fischer und der Landesregierung von Anfang an gespannt ${ }^{106}$. Dieser drängte für ihren Geschmack in der Entschädigungsfrage zu schnell vorwärts, während die württemberg-badische Landesregierung lieber eine Initative auf breiterer Basis abwarten wollte. Sie verwies darauf, daß sich „eine solche Wiedergutmachung nur im Rahmen der tatsächlich vorhandenen Möglichkeiten halten" ${ }^{107}$ könne und „zur Behandlung der Frage ... eine gesetzliche Grundlage notwendig“ sei, die

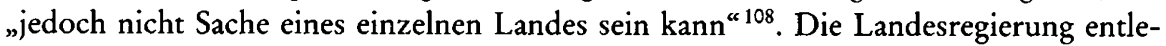
digte sich des Problems am 14. November durch die Entlassung Fischers und die Auflösung seines Amtes ${ }^{109}$. Die Dienststelle wurde zwar formell als Hauptabteilung IX des Innenministeriums weitergeführt, doch in der Praxis hieß das, daß die Belange der Verfolgten nunmehr weitgehend vernachlässigt blieben. Die bayerische Anregung beim Stuttgarter Länderrat, ebenfalls ein Staatskommissariat einzurichten, wies Ministerpräsident Reinhold Maier mit der Begründung zurück, daß Bayern ein wesentlich größeres Land sei: „Wir machen eine Dienststelle, aber keinen Staatskommissar. " 110 Demgemäß wurde in Württemberg-Baden zunächst auch keine eigene Betreuung für die Verfolgten aufgebaut, und die Landräte und Bürgermeister blieben neben der bescheidenen Dienststelle im Innenministerium die einzigen von Amts wegen damit befaßten staatlichen Repräsentanten.

Der Hauptbeitrag der Abteilung IX des Innenministeriums zugunsten der Opfer des Nationalsozialismus bestand darin, daß sie am 17. Dezember 1945 einen Rundbrief an die Landräte sowie die Oberbürgermeister in Stuttgart, Heilbronn und Ulm verschickte, in dem sie darauf hinwies, daß "den ehemaligen politischen Verfolgten ... eine besondere Betreuung zuteil werden" müsse. Das Rundschreiben empfahl die erwähn-

105 Vgl. Paul Sauer, Demokratischer Neubeginn in Not und Elend. Das Land Württemberg-Baden von 1945 bis 1952, Ulm 1978, S. 12, $23 \mathrm{ff}$.

106 Siehe etwa Franz Fischer an Ministerpräsident Reinhold Maier u. Staatsrat Konrad Wittwer, 10.11. 1945, BadWürtHStA, EA 1/920, Bü. 709.

107 Wittwer an Fischer, 23. 10. 1945, BadWürtHStA, EA 1/920, Bü. 709.

108 Wittwer an Fischer, 15. 11. 1945, BadWürtHStA, EA 1/920, Bü. 709.

109) Auszug aus der Niederschrift über die Sitzung des württemberg-badischen Staatsministeriunfs vom 14.11. 1945, BadWürtHStA, EA 1/920, Bü. 709.

110 Niederschrift der Sitzung des württemberg-badischen Staatsministeriums am 3.1. 1946, BadWürtHStA, EA 4/001, Bü. 57a. 
ten, in Stuttgart getroffenen Maßnahmen zur Nachahmung. Zugleich wurde in Aussicht gestellt, die dabei entstehenden Aufwendungen später ganz oder teilweise zu ersetzen ${ }^{111}$. Dagegen erhob sich aus dem Kreis der Verfolgten der Vorwurf, dies sei entschieden zu unverbindlich formuliert ${ }^{112}$.

Tatsächlich lag in den ersten beiden Jahren nach Kriegsende in Württemberg-Baden die Hauptlast der Betreuung bei den Organisationen der Verfolgten selbst. Nachdem ähnlich wie in Stuttgart ehemalige Verfolgte in vielen Kreisen Betreuungsstellen geschaffen hatten, wurde schließlich am 17. März 1946 mit Genehmigung der Militärregierung die Landesstelle für die politisch Verfolgten des Naziregimes gegründet, die als Dachorganisation für die zahlreichen Betreuungsstellen in Württemberg-Baden fungierte. Sie war Bestandteil des Landesausschusses der politisch Verfolgten. Im April 1947 ging der Landesausschuß auch in Württemberg-Baden in die Vereinigung der Verfolgten des Naziregimes (VVN) über, und zusammen mit ihm auch die Landesstelle. Für einige Zeit wuchs sie in eine geradezu halbamtliche Stellung hinein.

Die in der Landesstelle zusammengefaßten Betreuungsstellen übernahmen die schwierige Aufgabe festzulegen, wer überhaupt als politischer Verfolgter anzusehen sei, und stellten Ausweise aus, die zur Inanspruchnahme der für Verfolgte des Nationalsozialismus vorgesehenen Vorteile berechtigten. Bis November 1947 erkannte sie 9.710 Menschen als Verfolgte an, darunter als größte Gruppe 6.590 politisch Verfolgte sowie 1.656 rassisch und 366 religiös Verfolgte. Unter den rassisch Verfolgten befanden sich neben 927 Juden und 640 von den Nazis als "Judenmischlinge" Verfolgte auch 89 Zigeuner ${ }^{113}$. Diese Zahlen betrafen natürlich nur die außerhalb der UNRRA-Betreuung stehenden deutschen Verfolgten, weshalb hier auch die Zahl der Juden verhältnismäßig gering ist. Die jüdische Bevölkerung Badens und Württembergs war nach 1933 zum Teil emigriert, von denen, die dageblieben waren, wurde ein Großteil ermordet. So lebten beispielsweise 1946 in Stuttgart nur noch 300 Juden gegenüber 10.400 in der Zeit vor Hitler ${ }^{114}$.

Neben der Auswahl der Bewerber hatte die Landesstelle die Aufgabe, die Interessen notleidender Verfolgter gegenüber den Arbeits-, Wohnungs- und Versorgungsämtern zu vertreten, um die ihnen zustehenden Rechte bei der Zuweisung von Arbeitsplätzen, Wohnraum, Lebensmitteln und anderen wichtigen Versorgungsgütern durchzusetzen. Bei der medizinischen Betreuung der ehemaligen Verfolgten half auch hier vor allem die Süddeutsche Ärzte- und Sanitätshilfe der Centrale Sanitaire Suisse, die Lebensmittel und Medikamente aus der Schweiz bereitstellte und Erholungsheime für kranke Verfolgte unterhielt ${ }^{115}$.

Die Tätigkeit der Landesstelle ersparte dem Staat eigene Anstrengungen. Die württemberg-badische Regierung konnte sich so über längere Zeit hinweg „mit einem un-

111 Rundschreiben des württemberg-badischen Innenministeriums Nr. IX 439, 17.12. 1945, IfZ-Archiv, MF 260, OMGUS-WB 12/26-2/26.

112 Protokoll der Landeskonferenz Württemberg-Baden der politisch Verfolgten des Naziregimes am 17. 3. 1946 in Stuttgart (Anm. 103).

113 Vereinigung der Verfolgten des Naziregimes Württemberg-Baden, Statistical Report Regarding the Work of Examination and Recognition in the Month of November 1947, IfZ-Archiv, MF 260, OMGUS-WB 12/26-2/ 24.

114 Minutes of the Meeting of the Property Disposition Board, 17. 9. 1946, IfZ-Archiv, MF 260, OMGUS-Bico 11/13-1/16.

115 Protokoll der 1. Landes-Delegierten-Konferenz der VVN Württemberg-Baden in Stuttgart am 17.5. 1947, S. 5, VVN/BW-Archiv, Geschichte/Dokumente der VVN. 
verhältnismäßig kleinen Behördenapparat für die Wiedergutmachung "116 begnügen. Die Militärregierung betrachtete auch diese Entwicklung mit zwiespältigen Gefühlen. James H. Campbell, Chef der Public Welfare Branch in Württemberg-Baden, berichtete am 21. Mai 1946 an seine vorgesetzte Stelle in Berlin, daß er bei aller Sympathie für die Ziele dieser Organisation prinzipiell gegen Verfolgtenorganisationen sei, da sich diese höchstwahrscheinlich zu politischen Pressure-groups entwickeln würden. Überdies war er überzeugt, „daß sich durch ein vernünftiges, unparteilich durchgeführtes Regierungsprogramm ${ }^{\text {“117 }}$ derartige Anstrengungen der Verfolgtenorganisationen erübrigen würden. Doch davon war man in Württemberg-Baden auch nach Campbells Auffassung im Moment noch weit entfernt. Die staatlichen Anstrengungen bei der Umsetzung der in den Military Government Regulations festgelegten Forderungen zugunsten der ehemaligen Verfolgten qualifizierte er als ungenügend; nach den Beobachtungen seiner Stelle waren "die deutschen Behörden bei der Ausführung dieser Bestimmungen lax“ ${ }^{118}$. Deshalb schickte seine Abteilung am 23. Mai 1946 ein ausführliches Schreiben an die Wohlfahrtsabteilung des württemberg-badischen Innenministeriums, in welchem festgestellt wurde, „daß viele der Kreisbehörden entweder nicht wüßten oder mißachtet hätten“, daß die Military Government Regulations den deutschen Verfolgten des Nationalsozialismus dieselben Privilegien wie den United Nations Displaced Persons gewährten und die deutschen Wohlfahrtsämter dort angewiesen seien, diesen die entsprechenden Rechte einzuräumen. Um Zweifel auszuschließen, wurden die einschlägigen Verordnungen der Military Government Regulations noch einmal aufgeführt. Das Schreiben schloß mit dem ausdrücklichen Befehl, daß diese Anordnungen nach Buchstaben und Geist berücksichtigt werden müßten ${ }^{119}$. Auch im Stuttgarter Länderrat monierten die Amerikaner, noch immer sei keine praktische Hilfe für die Opfer des Nazismus geleistet worden; entsprechende deutsche Sofortmaßnahmen würden erwartet ${ }^{120}$.

Schließlich wies Innenminister Fritz Ulrich seine Regierung darauf hin, daß es nicht länger vertretbar sei, nur ein Referat seines Ministeriums beiläufig mit dieser Angelegenheit zu betrauen ${ }^{121}$. Im Juli wurde daraufhin dieses Referat ein Stück aufgewertet, indem wieder ein selbständiges „Amt für Wiedergutmachung“ unter der Leitung des Wirtschaftsministers Josef Andre gebildet wurde ${ }^{122}$. Doch handelte es sich auch hierbei noch um ein Provisorium, und erst Anfang 1947 kam es zu einer dauerhaften Lösung. Am 11. Februar 1947 beschloß die württemberg-badische Regierung nach längeren Beratungen, im Justizministerium eine Abteilung Wiedergutmachung einzurichten ${ }^{123}$. Leiter der neu ins Leben gerufenen Stelle wurde der bisherige Leiter der Gesetzgebungsabteilung des Justizministeriums, Otto Küster. Unter der Ägide des 1933 seines

116 Justizminister Beyerle an württemberg-badisches Staatsministerium, 7.3. 1947, BadWürtHStA, EA 1/920, Bü. 709.

117 James H. Campbell an Public Welfare Section, OMGUS, 21. 5. 1946, IfZ-Archiv, MF 260, OMGUS-CAD 5/323-3/6.

118 Ebenda.

119 William S. Fitzer an Buchmann, 23.5. 1946, IfZ-Archiv, MF 260, OMGUS 12/26-2/26.

120 Fritz Ulrich (württ.-bad. Innenminister) an württemberg-badisches Staatsministerium, 31.5. 1946, BadWürtHStA, EA 1/920, Bü. 709.

121 Ebenda.

122 Niederschrift über die Sitzung des württemberg-badischen Staatsministerium am 3.7. u. 10.7. 1946, BadWürtHStA, EA 4/001, Bü. 57 a.

123 Niederschrift über die Sitzung des württemberg-badischen Staatsministeriums vom 11.2. 1947, BadWürtHStA, EA 4/001, Bü. 57a. 
Richteramts enthobenen Küster, einem der geistigen Väter des konstruktiven Mißtrauensvotums ${ }^{124}$, gewann die württemberg-badische Wiedergutmachungspolitik bald erheblich an Profil.

Im Zuge dieser Entwicklung gingen auch hier die Kompetenzen für die Fürsorge von den Betreuungsstellen der Verfolgten auf staatliche Stellen über. In einem Erlaß vom 14. Juni 1947 wurde die Ernennung von öffentlichen Anwälten für die Wiedergutmachung in jeder Kreisstadt sowie die Errichtung jeweils einer Landesbezirksstelle für die Wiedergutmachung in Stuttgart und Karlsruhe angeordnet, die unter der Oberaufsicht der Wiedergutmachungsabteilung des Justizministeriums standen ${ }^{125}$. Das bedeutete, daß hilfsbedüftige Verfolgte sich nicht mehr wie bisher an das Sozialamt wenden mußten, sondern einen Antrag bei der Abteilung Wiedergutmachung des Justizministeriums stellen konnten ${ }^{126}$. Dies war ein wichtiger, psychologisch bedeutsamer Schritt in Richtung auf die Umwandlung der Fürsorgeberechtigung der Verfolgten des Nationalsozialismus in Rechtsansprüche.

c) Großhessen

In Großhessen entstanden gleichfalls bald nach Kriegsende in den meisten Stadt- und Landkreisen sogenannte „Sonderbetreuungsstellen“, die sich um die Opfer des NSRegimes kümmerten. Zum größten Teil wurden diese Betreuungsstellen von den Bezirksfürsorgeverbänden getragen, wobei zunächst mangels einheitlicher Direktiven jede Betreuungsstelle nach eigenem Gutdünken arbeitete. In den Städten unterstanden sie dem Oberbürgermeister, in den Landkreisen der Aufsicht des Landrats ${ }^{127}$. Daneben existierten aber auch in Großhessen Betreuungsstellen, die aus der Eigeninitiative der Verfolgten hervorgegangen waren und keinen Behördencharakter besaßen.

Seit 1. November 1945 bestand im großhessischen Innenministerium eine Abteilung Wiedergutmachung für politisch, rassisch und religiös Verfolgte, die im Januar 1947 dem Befreiungsministerium angegliedert wurde. Zum Leiter bestellte die großhessische Regierung Anfang April 1946 Curt Epstein, der wegen seines jüdischen Glaubens einige Jahre in einem $\mathrm{KZ}$ verbracht hatte ${ }^{128}$. Im Gegensatz zu Württemberg-Baden nahm die großhessische Landesregierung den bayerischen Vorschlag auf Einrichtung eines Staatskommissariats für die rassisch Verfolgten positiv auf. Chef der neuen Behörde wurde Epstein, der nun in Personalunion die Wiedergutmachungsabteilung des Innenministeriums und das Staatskommissariat für die Betreuung der Juden leitete ${ }^{129}$.

Bald zeigte sich in Großhessen dieselbe Entwicklung wie in Bayern und Württem-

${ }^{124}$ Vgl. Reinhold Maier, Ein Grundstein wird gelegt. Die Jahre 1945-1947, Tübingen 1964, S. 251 f.

125 Verordnung Nr. 162 des Staatsministeriums über den Aufbau der Wiedergutmachungsbehörden vom 14.6. 1947, in: Regierungsblatt der Militär-Regierung Württemberg-Baden, Nr. 9, 7.7. 1947, S. 57.

126 Rundschreiben des Innenministeriums, Abt. Wiedergutmachung, Nr. IX 779, an die Landratsämter von Nord-Württemberg und Bürgermeisterämter in Stuttgart, Heilbronn u. Ulm, 21./30.5. 1947, VVN/BWArchiv, Wiedergutmachung-Entschädigungsgesetz.

127 Curt Epstein an Ministerpräsident Karl Geiler, 22.7. 1946, HessHStA, Abt. 502, Nr. 2773b; Epstein an Sharon L. Hatch (OMGH), 5.6. 1946, IfZ-Archiv, MF 260, OMGUS 8/66-1/3.

128 Tätigkeitsbericht der Wiedergutmachungsabteilung für politisch und religiös Verfolgte beim Ministerium für politische Befreiung sowie des Staatskommissariates für die Betreuung der Juden in Hessen, 11.8. 1947, HessHStA, Abt. 502, Nr.2772c. Vgl. auch Wolf-Arno Kropat, Jüdische Gemeinden, Wiedergutmachung, Rechtsradikalismus und Antisemitismus nach 1945, in: Neunhundert Jahre Geschichte der Juden in Hessen. Beiträge zum politischen, wirtschaftlichen und kulturellen Leben, hrsg. v. d. Kommission für die Geschichte der Juden in Hessen, Wiesbaden 1983, S. 469 f.

129 Beschluß-Protokoll über die Sitzung des großhessischen Kabinetts am 4.4. 1946, HessHStA, Abt. 502, Nr. 4648. 
berg-Baden: Vor allem die Verfolgten selbst wünschten dringend aus dem System der allgemeinen Fürsorge herausgenommen zu werden und forderten eine von der öffentlichen Wohlfahrt unabhängige Betreuung ${ }^{130}$. Im September 1946 kam der hessische Ministerrat diesem Wunsch schließlich nach und verabschiedete eine Verordnung zur Vereinheitlichung der Betreuungsstellen, die diese zugleich aus dem System der allgemeinen Fürsorge ausgegliederte ${ }^{131}$. Nun entstanden in jedem Stadt- und Landkreis eine Betreuungsstelle sowie Hauptbetreuungsstellen in Kassel, Wiesbaden und Darmstadt, die dem Innenministerium unterstanden. Diese Ämter entschieden über die Aufnahme in den Kreis der Betreuten und über Art und Umfang der Betreuung. Die Verordnung bestimmte zugleich eine Reihe von Sofortmaßnahmen. Dazu gehörten die Beschaffung von Arbeitsplätzen, Bekleidung und Wohnraum, Vorschußzahlungen auf künftige Wiedergutmachungsleistungen bei Bedürftigkeit, die Zuweisung von Kleingärten, Rückübereignung von geraubten Vermögenswerten und Rechten, die Rückzahlung von Sühneabgaben an die Juden sowie die ärztliche Versorgung der Verfolgten ${ }^{132}$.

Ein Tätigkeitsbericht vom August 1947 schilderte, wie sich die Betreuungsstellen mit wechselndem Erfolg bemühten, diese Sofortmaßnahmen durchzuführen. So wurden in Zusammenarbeit mit dem Landeswirtschaftsamt dringend benötigte Kleidungsstücke wenigstens zum Teil beschafft und die Ernährung der Verfolgten durch Ausgabe von Lebensmittel-Zulagen aufgebessert. Besondere Schwierigkeiten traten auf, wenn es darum ging, den ehemaligen Verfolgten beim Wiederaufbau einer Existenz behilflich zu sein, sei es durch die Vergabe von Lizenzen, Gewerbescheinen, bei der Arbeits- und Wohnungsvermittlung oder auch der Möbelbeschaffung. Hier klagten die Verfolgten häufig über ablehnendes Verhalten der zuständigen Stellen. Mit Unterstützung der Centrale Suisse Sanitaire, die auch in Hessen bei der medizinischen Betreuung der ehemaligen Verfolgten tätig war, konnte in Bad Salzhausen ein Erholungsheim für ehemalige KZ-Häftlinge eingerichtet werden. Außerdem wurde 120 Studenten aus dem Verfolgtenkreis durch Stipendien ein Studium ermöglicht. Darüber hinaus unterstützte das Staatskommissariat den Wiederaufbau des jüdischen Lebens etwa durch Wiederherstellung von Friedhöfen, Synagogen sowie Sozialeinrichtungen ${ }^{133}$. Alles in allem ähnelten die in Hessen durchgeführten Unterstützungsmaßnahmen weitgehend denen in Bayern und Württemberg-Baden. Die Zahl der hier betreuten Verfolgten lag dabei etwa in der Größenordnung Württemberg-Badens. Mitte 1947 existierten 48 großhessische Betreuungsstellen in den Stadt- und Landkreisen, die 10.487 Verfolgte betreuten. Davon waren 5.439 politisch, 3.564 rassisch und 326 religiös Verfolgte. Hinzu kamen noch 1.157 gleichfalls betreute Hinterbliebene ${ }^{134}$.

130 Die Vorsitzenden der Betreuungsstellen von Hanau, Wiesbaden, Giessen, Fulda, Darmstadt, Offenbach, Kassel, Frankfurt a.M. an das großhessische Innenministerium, 10.8. 1946, HessHStA, Abt. 502, Nr. 2772c.

131 Verordnung zur Vereinheitlichung der Betreuungsstellen in Groß-Hessen, 11.9. 1946, (am 27.11. in Kraft getreten), BadWürtHStA, EA 4/001, Bü. 35.

132 Ebenda, $\$ 5$.

133 Tätigkeitsbericht der Wiedergutmachungsabteilung vom 11.8. 1947 (Anm. 128).

134 Ebenda. 


\section{Abgrenzung und Ausgrenzung von Verfolgten bei der Betreuung}

Die vorübergehende Besserstellung der Verfolgten im Rahmen einer erweiterten Fürsorge stieß, wie immer wieder von Verfolgtenseite beklagt wurde, in der deutschen Bevölkerung vielfach auf Ablehnung ${ }^{135}$. Auch manche Behörden förderten die verbreitete Auffassung, daß die allgemeinen Versorgungsschwierigkeiten auf die Privilegien der Verfolgten zurückzuführen seien. Karl Hauff, Leiter der württemberg-badischen Landesstelle der politisch Verfolgten, appellierte deshalb am 12. Februar 1947 in einer Rundfunkansprache über Radio Stuttgart an die Bevölkerung, daß die angeordnete Bevorzugung „in Wirklichkeit keine Bevorteilung gegenüber der übrigen Bevölkerung, sondern nur eine Angleichung" bedeute ${ }^{136}$. Derartige Einstellungen basierten zum Teil auf schlichtem Neid. Doch kam hinzu, daß die „diffamierende Taktik des Nationalsozialismus, dem Ausspruch eines Göring gerecht zu werden, daß die KZ-Lager die Dreckeimer der Nation seien " ${ }^{137}$, nicht ohne Wirkung geblieben war. Die Inhaftierung zahlreicher "gewöhnlicher" Krimineller in den Konzentrationslagern vor allem in der zweiten Hälfte des sogenannten Dritten Reiches hatte dazu beigetragen, daß deren Insassen im breiten Bewußtsein der Bevölkerung pauschal als Verbrecher gegolten hatten, und diese Bewertung wirkte auch nach dem Ende der nationalsozialistischen Herrschaft noch kräftig nach ${ }^{138}$.

Um dieser Stigmatisierung zu entkommen, verfochten vor allem die politisch Verfolgten eine scharfe Abgrenzung unter den ehemaligen KZ-Häftlingen. In seiner Begrüßungsansprache auf der 1. Landeskonferenz der politisch Verfolgten in WürttembergBaden in Stuttgart am 17. März 1946 erklärte das Vorstandsmitglied Karl Keim: „Außerdem ist da noch ein Unterschied zwischen KZlern und KZlern. Wir haben schon im Lager nicht nur gegen die SS gekämpft, sondern auch gegen die Kriminellen, die sogenannten Grünen“139. Und hessische politisch Verfolgte erklärten: „Asoziale und kriminelle Elemente schädigen unser Ansehen. Wir haben es nicht verdient, daß man uns in einem Atemzug mit diesen Elementen nennt. “140 Karl Hauff, der Leiter der württemberg-badischen Landesstelle, versuchte auch der Militärregierung klarzumachen, warum ein Unterschied zwischen den KZ-Häftlingen zu machen sei: Bis 1939 seien die KZInsassen in erster Linie politisch Verfolgte gewesen. Nach dieser Zeit seien echte Kriminelle, Geistesschwache und -kranke sowie andere sozial unerwünschte Personen in die Konzentrationslager geworfen worden. Diese Leute seien nicht würdig für den Verfolgtenstatus ${ }^{141}$. Deshalb gehörte es zu den Hauptaufgaben der Betreuungsstellen bzw. der Staatskommissariate in der US-Zone, die sogenannten kriminellen Häftlinge auszu-

135 Dr. Walter an Political Affairs, OMGB-ICD, 6. 8. 1946, IfZ-Archiv, MF 260, OMGUS 10/109-2/2.

136 Karl Hauff, Vortrag über die Lage der politisch Verfolgten, 12.2. 1947, IfZ-Archiv, MF 260, OMGUS-WB 12/26-2/24.

137 Karl Keim, in: Protokoll der 1. Landesdelegiertenkonferenz der VVN, 17.5. 1947 (Anm. 115).

138 Vgl. etwa Barbara Distel, "Asoziale und Berufsverbrecher", in: Legenden - Lügen - Vorurteile. Ein Lexikon zur Zeitgeschichte, hrsg. v. Wolfgang Benz, München 1990, S. 28-31.

139 Karl Keim, in: Protokoll der Landeskonferenz Württemberg-Baden der politisch Verfolgten des Naziregimes, 17.3. 1946 (Anm. 103). Vgl. zu diesem Problem auch Falk Pingel, Häftlinge unter SS-Herrschaft. Widerstand, Selbstbehauptung und Vernichtung im Konzentrationslager, Hamburg 1978, S. 102-117.

140 Die Vorsitzenden der Betreuungsstellen von Hanau usw. an das großhessische Innenministerium, 10. 8. 1946 (Anm. 130).

141 Tagebucheintrag der Public Welfare Branch, OMGWB, vom 10.7. 1946, IfZ-Archiv, MF 260, OMGUS-WB 12/26-2/26. Vgl. zu diesem Problem auch Pingel, Häftlinge unter SS-Herrschaft, S. $69 \mathrm{ff}$. 
sieben ${ }^{142}$. In Stuttgart arbeiteten die Betreuungsstellen dabei mit einer eigens eingerichteten KZ-Prüfstelle der Polizei zusammen, in der auch ehemalige KZ-Häftlinge mitwirkten $^{143}$. Insgesamt fielen bei der Auswahl etwa die Hälfte der Antragsteller durch ${ }^{144}$. Unter den abgelehnten Bewerbern war auch Hjalmar Schacht, der sich Ende 1946 in Stuttgart vergeblich um den Status eines Verfolgten des Nationalsozialismus bemühte ${ }^{145}$.

Die strikte Abgrenzung von den sogenannten kriminellen Häftlingen war zum einen natürlich eine Folge des von der SS praktizierten raffinierten Unterdrückungsystems in den Konzentrationslagern, wobei sie sich vielerorts krimineller Häftlinge zur Terrorisierung und Kontrolle der übrigen Gefangenen bedient hatte ${ }^{146}$. Somit lassen sich diese Abgrenzungsbemühungen vielfach aus diesen leidvollen Erfahrungen erklären. $\mathrm{Zu}$ gleich wird hier aber auch deutlich, daß die in nationalsozialistischer Zeit gebräuchlichen Kategorien der "Asozialität" oder des "gemeinschaftsschädlichen Verhaltens“ noch nachwirkten ${ }^{147}$. Man kann also feststellen, daß in den ersten Nachkriegsjahren auch unter den rassisch, religiös und politisch Verfolgten die Kriminalisierung mancher normabweichender moralischer und sozialer Standards in der NS-Zeit nicht in Frage gestellt wurde, allenfalls wurde das übermäßige Strafmaß kritisiert. Dies läßt darauf schließen, daß die Verbreitung mancher Einstellungen und Vorurteilsstrukturen, die dabei eine Rolle gespielt hatten, quer durch alle Schichten der deutschen Bevölkerung verlief und sich nicht auf den dezidiert nationalsozialistisch eingestellten Teil beschränkte.

Doch lag den Bemühungen der politisch Verfolgten auch die Absicht zugrunde, ein Leitbild des politisch bewußten antifaschistischen Kämpfers zu schaffen, der sich einerseits von den „Nur-Opfern“, zu denen auch etwa die Juden gehörten, und andererseits von den „Kriminellen und Asozialen“ abhob. Die Sicht der ausländischen jüdischen Organisationen wurde hier also gerade umgedreht. Nicht die Juden, sondern die politisch Verfolgten seien die ersten Opfer der Nationalsozialisten gewesen. Da unter den in Deutschland überlebenden deutschen Opfern des NS-Regimes die politisch Verfolgten zunächst den Hauptanteil stellten - die meisten Juden in Deutschland waren ausländischer Herkunft und fielen nicht in die Zuständigkeit der Betreuungsstellen - besaßen derartige Auffassungen natürlich anfänglich besonderes Gewicht.

Dies schlug sich auch in den Auswahlkriterien für die Aufnahme in die Verfolgtenbetreuung durch die KZ-Betreuungsstellen in der US-Zone nieder, die bis zur endgültigen gesetzlichen Regelung Mitte 1947 galten. So teilten die KZ-Betreuungsstellen in Würt-

142 Siche beispielsweise auch Otto Aster, Bericht über die Betreuung der politisch Verfolgten in Bayern, 21. 10. 1946, IfZ-Archiv, NL Hoegner, ED 120, Bd. 116.

$143 \mathrm{Karl}$ Keim, in: Protokoll der 1. Landeskonferenz Württemberg-Baden der politisch Verfolgten des Naziregimes am 17.3. 1946 (Anm. 103).

144 Tagebucheintrag der Public Welfare Branch, OMGWB, 10.7. 1946, IfZ-Archiv, MF 260, OMGUS-WB 12/ 26-2/26.

145 Tagebucheintrag der Public Welfare Branch, OMGWB, 6. 12. 1946, IfZ-Archiv, MF 260, OMGUS-WB 12/ 26-2/26.

146 Vgl. dazu etwa Pingel, Häftlinge unter SS-Herrschaft, S. 102-117; Hermann Langbein, ... nicht wie die Schafe zur Schlachtbank. Widerstand in den nationalsozialistischen Konzentrationslagern 1938-1945, Frankfurt a.M. 1980, S.44-56.

147 So forderte etwa der Bayerische Gewerkschaftsbund am 17.1. 1948 von der bayerischen Staatsregierung unter anderem die „Einweisung aller asozialen Elemente in Arbeitslager“. Vgl. Paul Erker, Solidarität und Selbsthilfe. Die Arbeiterschaft in der Ernährungskrise, in: Neuanfang in Bayern 1945-1949. Politik und Gesellschaft in der Nachkriegszeit, hrsg. v. Wolfgang Benz, München 1988, S. 96. 
temberg-Baden, wo die Eigeninitiative der Verfolgten aufgrund der anfänglichen Zurückhaltung des Landes eine besondere Rolle spielte, zwei Arten von Ausweisen aus. Die erste Ausweiskategorie galt für politische Überzeugungstäter, „die bewußt und gewollt in den meisten Fällen im organisierten Zusammenhang mit Gleichgesinnten aus politischen Gründen ... Widerstand leisteten“. Die zweite galt für politische Gelegenheitstäter, Verfolgte aus religiösen, weltanschaulichen oder rassischen Gründen, Emigranten, Personen aus der Illegalität, Hinterbliebene und Sonderfälle. Unter die letzte Rubrik fielen etwa Fälle von Wehrkraftzersetzung, Spionage, früherer Mitgliedschaft in einer militärischen Organisation oder der NSDAP, sofern egoistische Motive ausfielen bzw. eine Umkehr zum aktiven Kampf stattgefunden habe. Ausländer aus Staaten der Vereinten Nationen wurden hingegen generell an die UNRRA verwiesen ${ }^{148}$. Auch in Bayern und Großhessen wurden ähnliche Unterscheidungen getroffen, wenngleich sich die Anerkennungskriterien im Detail unterschieden ${ }^{149}$. Den zuständigen Militärregierungsstellen erschienen derartige Auslegungen der von ihr vorgegebenen Kriterien hin und wieder suspekt, so daß sie schließlich im Zusammenhang der Entschädigungsgesetzgebung auf eine genaue Klärung der Definition von nationalsozialistischer Verfolgung drängen sollten ${ }^{150}$.

Die Verbindung von erlittener Verfolgung und persönlicher Haltung der Verfolgten führte dabei vielfach zu Schwierigkeiten. So gründeten ehemalige als kriminell oder asozial inhaftierte Häftlinge, worunter auch die Opfer der sogenannten "vorbeugenden Verbrechensbekämpfung “ fielen ${ }^{151}$, eine eigene Verfolgtenvereinigung mit dem Namen „Die Vergessenen“, die für diese Gruppe die Anerkennung und Betreuung als Verfolgte des Nationalsozialismus anstrebte ${ }^{152}$. Der bayerische Innenminister Josef Seifried förderte Anfang 1947 einen Gesetzentwurf über die Fürsorge für übermäßig bestrafte, nicht-politische Häftlinge ${ }^{153}$. Finanzstaatssekretär Richard Ringelmann verwahrte sich jedoch brüsk dagegen, daß diesen Personen über die öffentliche Fürsorge hinausgehende Hilfe geleistet würde, solange die dem Staat zur Verfügung stehenden Mittel nicht ausreichten, „um unbescholtenen Totalfliegergeschädigten, Ausgewiesenen und Flüchtlingen " entsprechende Hilfe zuteil werden zu lassen ${ }^{154}$. Bei der ganzen Frage der Fürsorge für die Opfer des Nationalsozialismus darf man also nicht aus den Augen verlieren, daß sich diese stets in scharfer Konkurrenz zu Ansprüchen anderer geschädigter Gruppen befanden.

Doch auch rassisch Verfolgte klagten über Diskriminierungen. So beschwerte sich der „vorbereitende Ausschuß für eine Hilfsstelle Rasseverfolgter nichtjüdischen Glaubens", daß insbesondere nichtjüdische Familienangehörige von Juden in Großhessen nicht ausreichend von den Betreuungsstellen unterstützt würden. Der Ausschuß ver-

148 Vereinigung der politischen Gefangenen und Verfolgten des Nazisystems, Richtlinien für die Betreuung der "Opfer des Faschismus“, 8. 1. 1946, IfZ-Archiv, MF 260, OMGUS-WB 12/26-2/26.

149 Richtlinien für den landeseinheitlichen Ausweis der ehemals politisch Inhaftierten, in: Mitteilungsblatt des Landesausschusses der politisch Verfolgten in Bayern, Nr. 3, 1.2. 1947; Richtlinien zur Durchführung der Verordnung über die Bildung und das Verfahren der Betreuungsstellen in Hessen vom 27.11. 1946 und vom 24. 3. 1947, HessHStA, Abt. 502, Nr. 2773a.

150 Vgl. Drittes Kapitel, Abschnitt III. 1.

151 Auerbach an bayerische Staatskanzlei, 6.1. 1949, BayHStA, MA 114263.

152 Niederschrift über die Sitzung von Vertretern der württemberg-badischen und bayerischen Betreuungsstellen am 19.7. 1946, VVN/BW-Archiv, Wiedergutmachungsbehörden (Sonderfondsgesetz).

153 Entwurf für ein Gesctz über die Fürsorge für übermäßig bestrafte nichtpolitische Häftlinge, 27. 1. 1947, BayHStA, MF 69410.

154 Ringelmann an Justizminister Hoegner, 20.6. 1947, BayHStA, MF 69410. 
wahrte sich dagegen, daß die Betreuung an den Nachweis bestimmter Verfolgungsmaßnahmen wie insbesondere eine längere KZ-Haft gebunden war. Demgegenüber forderte er, Rücksicht darauf zu nehmen, „daß hier nicht allein ausschlaggebend ist, was nun tatsächlich von dem Einzelnen erduldet wurde, sondern auch, was noch bevorstand“ ${ }^{155}$. Der großhessische Staatskommissar Epstein reagierte erbost auf diese Vorhaltungen. Für diesen Personenkreis müßten dieselben Kriterien gelten wie für die anderen Verfolgten. Insbesondere legte Epstein Wert darauf, daß die Zuerkennung der Verfolgteneigenschaft auch von einer entsprechenden Haltung abhängig sei, wobei er einem Teil des betreffenden Kreises vorwarf, es daran fehlen gelassen zu haben. Es käme nicht in Frage, „Personen ...., die sich in der kritischen Zeit des Nationalsozialismus möglichst weit von Juden und anderen Verfolgten distanzierten“, zu betreuen. Für „politische Wetterfahnen“ sei „kein Platz in den Reihen der politisch, rassisch und religiös Verfolgten ..., die für ein besseres, ganz besonders demokratisches Deutschland gekämpft, gelitten und geblutet haben" ${ }^{156}$. Die hessische Regierung schloß sich schließlich dem Standpunkt Epsteins an, daß „ein Unterschied in der Betreuung je nach der erlittenen Verfolgung gemacht werden “ ${ }^{157}$ müsse. Damit waren Betroffene der Nürnberger Gesetze - anders als die jüdischen Verfolgten - nicht als Gruppenverfolgte, sondern nur nach Maßgabe ihrer individuellen Verfolgung anspruchsberechtigt.

Von Anfang an gab es auch besondere Probleme bei den verfolgten Zigeunern. Diese wurden beispielsweise in den großhessischen Richtlinien ausdrücklich unter den rassisch Verfolgten, die dort einen Betreuungsanspruch zugesprochen bekamen, aufgeführt, allerdings nur, „sofern sie heute einen geregelten Beruf ausüben und einen festen Wohnsitz haben “158. Damit forderten aber diese Bestimmungen, die unter anderem gerade die Versorgung mit Wohnungen und Arbeit für Verfolgte regeln sollten, von dieser Gruppe als Voraussetzung, daß sie über eben jene Attribute einer geregelten Existenz bereits verfügte. Andererseits wurde Zigeunern bei den Ämtern weiterhin häufig die Türe gewiesen, wie ein bewegender Hilferuf der Betreuungsstelle Marburg zugunsten obdachloser Zigeuner vom Sommer 1947 schilderte: „Wohnraum wird ihnen von den Wohnungsämtern nicht zugewiesen, da sie angeblich asozial angehaucht sind ... Ein anständiges und ordnungsmäßiges Leben wird diesen Menschen bis heute noch nicht ermöglicht, da sie von den Polizeiorganen ständig von einem Ort zum anderen getrieben werden. " 159 Die nationalsozialistische Diskriminierung konnte also auch in der Betreuung der ehemaligen Verfolgten weiterwirken, sofern diese auf Traditionen beruhte, die einen außernationalsozialistischen Hintergrund besaßen und deshalb nicht zwangsläufig diskreditiert waren.

155 Der vorbereitende Ausschuß für eine Hilfsstelle Rasseverfolgter nichtjüdischen Glaubens an Ministerpräsident Geiler, 30.9. 1946, HessHStA, Abt. 502, Nr. 2773a.

156 Epstein an Gottlob Binder (hess. Minister für politische Befreiung), 19.11. 1946, HessHStA, Abt. 502, Nr. 2773a.

157 Hilpert an die Hilfsstelle für rassisch verfolgte Christen, 20.12. 1946, HessHStA, Abt. 502, Nr. 2773a.

158 Richtlinien zur Durchführung der Verordnung über die Bildung und das Verfahren der Betreuungsstellen in Hessen vom 27. 11. 1946 und vom 24.3. 1947 (Anm. 149). Die Bindung der Verfolgtenbetreuung für Zigeuner an ihre Seßhaftigkeit war in der US-Zone allgemein üblich. Vgl. etwa "Richtlinien für den landeseinheitlichen Ausweis der ehemaligen politischen Inhaftierten“, in: Mitteilungsblatt des Landesausschusses der politisch Verfolgten, Nr. 3, 1.2. 1947.

159 Betreuungsstelle für rassisch, religiös und politisch Verfolgte Marburg, 12.9. 1947, IfZ-Archiv, MF 260, OMGUS 8/66-2/3. 